\title{
PLANT COMMUNITIES WITH YELLOW OAT GRASS (TRISETUM FLAVESCENS (L.) PB.) IN THE SUBMONTANE AND MONTANE REGIONS OF SLOVENIA
}

\author{
Tina PETRAS SACKL ${ }^{1}$, Mitja KALIGARIC̆ ${ }^{2}$, Danijel IVAJNŠIC̆ ${ }^{2} \&$ \\ Sonja ŠKORNIK ${ }^{2}$
}

\begin{abstract}
In the present study, we investigated plant communities with Yellow Oat Grass (Trisetum flavescens (L.) $\mathrm{Pb}$.) in the submontane and montane regions of Slovenia. In 2005-2007 ninety-one relevés were collected by using the standard procedure of the Braun-Blanquet approach. Relevés were analysed with multivariate analysis and classified within two associations: Astrantio-Trisetetum (Polygono-Trisetion) and the Pastinaco-Arrhenatheretum (Arrhenatherion). Management practices, soil conditions and altitude were found to be significant factors for a further subdivision of both associations. Within the Astrantio-Trisetetum association three subassociations could be distinguished: -typicum, -buphthalmetosum and -trollietosum, and subassociations -typicum, -medicagetosum lupulinae, as well as -lolietosum subass. nova in the Pastinaco-Arrhenatheretum. The floristic composition and ecological characteristics of these plant communities are described and their implications for grassland conservation in Slovenia are discussed.
\end{abstract}

Key words: vegetation, anthropogenous grasslands, Astrantio-Trisetetum, Pastinaco-Arrhenatheretum.

Izvleček

V članku predstavljamo rezultate raziskave vegetacije travišč s prevladujočim rumenkastim ovsencem (Trisetum flavescens) v submontanskih in montanskih predelih Slovenije. V letih 2005-2007 smo popisali 91 vegetacijskih sestojev po standardni Braun-Blanquetovi metodi. Na osnovi multivariatnih analiz smo uvrstili travišča v dve asociaciji, in sicer v asociacijo Astrantio-Trisetetum (zveza Polygono-Trisetion) in asociacijo Pastinaco-Arrhenatheretum (zveza Arrhenatherion). Nadaljnja členitev na nižje sintaksonomske enote obeh asociacij je pogojena predvsem s stopnjo intenzivnosti upravljanja s travišči, z različnimi edafskimi razmerami in nadmorsko višino. Tako ločimo v asociaciji Astrantio-Trisetetum naslednje sintaksone: subasociacije -typicum, -buphthalmetosum in -trollietosum, v asociaciji Pastinaco-Arrhenatheretum pa: -typicum, -medicagetosum lupulinae in -lolietosum subass. nova. Predstavljene so floristična sestava asociacij, njune ekološke značilnosti in varstveni vidiki obravnavanih travišč.

Ključne besede: vegetacija, antropogena travišča, Astrantio-Trisetetum, Pastinaco-Arrhenatheretum.

\section{INTRODUCTION}

While almost half of Slovenia is covered by forests, grasslands constitute only $28.3 \%$ of the total area (Kladnik \& Gabrovec 1998). Despite their smaller area with extensive management, natural and anthropogenic grasslands constitute one of the most important sources for landscape biodiversity (Gomez-Pompa \& Kaus 1992, Swift et al. 1996). In addition, grasslands are invaluable sources of genetic diversity, provide habitats for numerous plant and animal species, and function as essential corridors between different habitat types. Anthropogenic grassland habitats, which have been known in Europe since the last millennium (Pärtel et al. 2006), further constitute an integral element in Slovenia's cultural heritage.

\footnotetext{
${ }^{1}$ Balos 7, 4290 Tržič, Slovenia, E-mail: tina.petras@gmail.com

${ }^{2}$ University of Maribor, Faculty of Natural Sciences and Mathematics, Department of Biology, SI-2000 Maribor, Koroška 160, Slovenia
} 
During recent last decades, because of the increasing use of fertilizers and the greater frequency of annual cuttings, many formerly extensively managed meadows of high biodiversity have been transformed into largely uniform grassland areas, dominated by the few competitive species that cope better in intensively managed agricultural environments (McKinney \& Lockwood 1999, Walker 2004). Particularly in the lowlands of Slovenia, habitat fragmentation constitutes an additional reason for the continuous degradation and disappearance of natural and semi-natural grassland ecosystems. Besides the erosion of plant species diversity, the decline of floristically rich meadows and pasturelands is the main reason for the decline of many farmland birds and other animals (Wilson et al. 2009). Consequently, the decrease in anthropogenic grassland habitats has recently stimulated a number of studies by phytoecologists (Swift et al. 1996, Walker et al. 2004, Pärtel et al. 2006) and animal ecologists (McCracken \& Tallowin 2004, Atkinson et al. 2004, Wilson et al. 2009).

The larger portion of anthropogenic grassland in Slovenia belongs to the class MolinioArrhenatheretea, which according to altitude and management, is further subdivided into five orders (Ellmauer \& Mucina 1993, Dierschke 2002). Some of the anthropogenic meadows and pasturelands of the upper montane belt of the Slovene Alps are classified within the Polygono-Trisetion alliance. These Trisetum flavescens dominated mesotrophic to eutrophic types of grassland are associated with humid, deeper soils, slightly acid to basic. They are widespread throughout the montane region of Central Europe (Ellmauer \& Mucina 1993, Ellenberg 1996, Merz 2002). Extensively managed grasslands of the Polygono-Trisetion alliance are rich in plant species (Ellmauer \& Mucina 1993). According to their floristical composition, meadows of the Polygono-Trisetion alliance represent the transition between the meadows that are characteristic of the lowland and the hay meadows of the montane region (Oberdorfer 1983). At lower altitudes Polygono-Trisetion meadows are replaced by Arrhenatherion grasslands of the order Arrhenatheretalia (Mucina 1993). The latter, more intensively managed grasslands, which tend to be associated with deeper, humid soils, are widely distributed in Central Europe (Oberdorfer 1983, Ellmauer \& Mucina 1993). Particularly high species diversity occurs in Arrhenatherion grasslands with Salvia pratensis, which are at the same time one of the most endangered plant communities of this alliance (Pott 1995, Ellenberg 1996). According to syntaxonomic aspects, they show a continuous transition into Bromion erecti-, Phyteumo-Trisetion- and Cynosurion-meadows (Ellmauer \& Mucina 1993).

The aim of the present study was to investigate the vegetation communities associated with Yellow Oat Grass (Trisetum flavescens) in the submontane and montane altitudinal belt of Slovenia. We hypothesized that (1) according to altitude, soil conditions and management, hay meadows with Trisetum flavescens may form different associations of the Polygono-Trisetion and Arrhenatherion alliances in which (2) due to lower nutrient input and the occurrence of plant species which are normally associated with natural subalpine meadows, species diversity was expected to increase with altitude.

To study the syntaxonomic characteristics and ecological conditions of yellow oat grass plant communities, vegetation samples were taken across the transition zone of the Polygono-Trisetion and Arrhenatherion alliance between $400 \mathrm{~m}$ and $1500 \mathrm{~m}$ a.s.l. Besides yielding of its geographical distribution, a better understanding of the environmental factors responsible for the formation of different associations will help in assessing the current conservation status of this vegetation type in Slovenia.

\section{METHODS}

\section{STUDY AREA}

Relevés of hay meadows rich with Trisetum flavescens were collected in the submontane and montane parts of Slovenia between $400 \mathrm{~m}$ and $1500 \mathrm{~m}$ a.s.l. Because of regionally prevailing patterns of land-use and the varying different altitudes, most samples were collected in the Alpine and pre-Alpine region, although grasslands with Yellow Oat Grass have also been found in the Dinaric region (Figure 1).

The Alpine region occupies most of the northern and central parts of Slovenia. To the south it borders the Dinaric region which represents the most northerly part of the Dinaric Mountains. The Dinaric region covers most of the southern part of Slovenia. Both regions are characterised by the prevalence of carbonate rocks, i.e. limestone and dolomite (Kladnik 1998, 1998a). Only 
the Pohorje and Kozjak Mountains (Central Alps) are mainly composed of metamorphic, silicate rocks (Žiberna 1998). With a mean altitude and inclination of $732 \mathrm{~m}$ a.s.l. and $18^{\circ}$, respectively, the highest altitudes and largest inclinations in Slovenia are found in the Alpine region. The relief of the Alps is very diverse, and the high variation in altitude between valley bottoms and the higher peaks has a significant influence on local climates. In the Alps two types of climate prevail: a montane and a moderate continental climate. In the submontane and montane belt of the mountains, annual mean temperatures vary between $8^{\circ}$ $\mathrm{C}$ and $10^{\circ} \mathrm{C}$, while the annual mean air temperature exceeds $10^{\circ} \mathrm{C}$ only at the bottom of the Soča river valley between Kobarid and Tolmin. In the Alpine region the annual amount of precipitation increases with altitude and from east to west. The Julian Alps receive $3000 \mathrm{~mm}$, higher altitudes in the Kamniško-Savinjske Alpe $2500 \mathrm{~mm}$, and the western parts of the Karavanke Mountains < $2000 \mathrm{~mm}$ of annual precipitation, while at higher altitudes in the Pohorje Mountains, in the far east of the Alps, annual precipitations can be as high as $1500 \mathrm{~mm}$. The Dinaric region is characterised by extensive high-altitude plateaus and lower land surrounded by higher mountains (podolja). On the high Dinaric plateaus, mean temperatures range between $6^{\circ} \mathrm{C}$ and $8^{\circ} \mathrm{C}$. While overall annual precipitation amounts to approximately $1100 \mathrm{~mm}$, the southern slopes of the Dinaric plateaus receive $>3000 \mathrm{~mm}$ of annual precipitation (Kladnik 1998, 1998a).

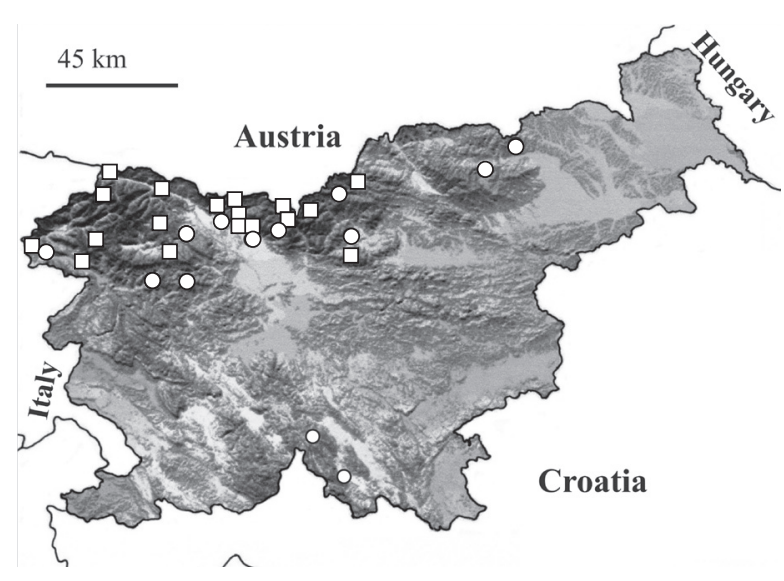

Figure 1: Map of occurence Astrantio-Trisetetum association $(\square)$ and Pastinaco-Arrhenatheretum associations ( $\circ$ ) in investigated area.

Slika 1: Karta pojavljanja asociacij Astrantio-Trisetetum ( $\square$ ) in Pastinaco-Arrhenatheretum ( $($ ) na območju popisovanja.

\section{Sampling methods and statistical analyses}

In 2005, 2006 and 2007, 91 relevés of grasslands with $\mathcal{T}$. flavescens were collected between June and early August. These were compiled by using the standard procedure of the Braun-Blanquet approach (Braun-Blanquet 1964, Westhoff \& van der Maarel 1973, Dierschke 1994).

For all 91 study plots (relevés), the following abiotic parameters were sampled: (1) location, (2) altitude ( $m$ a.s.l.), (3) exposure, (4) inclination $\left({ }^{\circ}\right)$, (5) geographic coordinates (subsequently, based on locations noted in the field), (6) pedologic units, and (7) number of species. Additionally some abiotic variables were estimated by weighted (species frequencies are weights) averages of Ellenberg indicator values (Ellenberg et al. 1991): (1) temperature; (2) moisture; (3) soil reaction $(\mathrm{pH})$ and (4) nutrient availability.

Additionally, 98 referential relevés of the studied grasslands from Slovenia (Čarni 2001: tab.1, relevés 8-18), Croatia (Horvatić \& Tomažič 1941: relevés 1, and 3-6), Austria (Aichinger 1933: tab. 18, relevés 2-8, Steinbuch 1995, tab.13, relevés 14, 704, 584, 87, 191, 137, 212, 700, 9317, 69, 380, 9128, 16, 39, 40), Germany (Machold 1991: tab. III.1, relevés 1-10, Oberforster 1986: p. 102-104, relevés $26,1,20,23,24,44,57,81,173,177,101,113$, 132, 139, 184, 205, 69, 87, 95, 116, Dierschke 1994: p. 188-190) and Italy (Poldini \& Oriolo 1994: tab. 7 , relevés 1-14) were collected and included in the analysis.

To classify Yellow Oat Grass grasslands according to their species composition, the species data set was composed of the 189 relevés (91 of our own relevés and the 98 referencial relevés from the literature). Braun-Blanquet cover-abundance data for the species were converted into a 2 to 9 scale (van der Maarel 1979). To differentiate the main associations of grasslands in which T. flavescens grows, this matrix was subjected to divisive clustering - Two Way Indicator Species Analysis (TWINSPAN; Hill 1979) using WinTWINS version 2.3 (Hill \& Šmilauer 2005). Additionally, we applied the ordination method - Principle Component Analysis (PCA) (Goodal 1954) to differentiate the subassociations within the recognized associations. Detrended Correspondence Analysis (DCA) (Hill \& Gauch 1980) was used to estimate the heterogeneity in the species data of our 91 relevés. Gradient length for the first DCA axis was 2.126, indicating that the linear ordination methods were suitable for the 
analysis. To relate the species composition of our 91 relevés to abiotic variables, Redundancy Discriminant Analysis (RDA) (van den Wollenberg 1977, ter Braak 2004) was used.

To test whether abiotic variables were significantly related to species composition, we used the Monte Carlo permutation test (499 permutations). The effect of rare species was reduced by downweighting. The ordination methods (PCA, DCA, RDA) and visualization of these results were carried out using the Canoco and Cano Draw programs (ter Braak \& Šmilauer 2002).

Geo-elements were determined according to Poldini (1991), and Raunkiaer's life forms according to Ellenberg \& Mueller-Dombois (1967) and Poldini $(1989,1991)$.

\section{NOMEnClature}

Taxonomic nomenclature follows Martinčič et al. (2007) and syntaxonomic nomenclature follows Ellmauer \& Mucina (1993), Knapp \& Knapp (1952), and Steinbuch (1995).

\section{RESULTS AND DISCUSSION}

\section{VEGETATION CLASSIFICATION}

On the basis of the Twinspan classification, 189 relevés of grasslands with $\mathcal{T}$. flavescens were defined as four main vegetation types - associations: Astrantio-Trisetetum Knapp et Knapp 1952, Pastinaco-Arrhenatheretum Passarge 1964, Centaureo transalpinae-Trisetetum Poldini et Oriolo 1994, and Lolio perennis-Cynosuretum Br-Bl. et De Leeuw 1936. While all our 95 relevés were classified within clusters representing the first two associations, the group (cluster) defined as the Lolio-Cynosuretum association included only referencial relevés and was not included in further analysis.

Syntaxonomical scheme:

Class Molinio-Arrhenatheretea R. Tx. 1937 em. R. Tx. 1970

Order Poo alpinae-Trisetetalia Ellmauer et Mucina 1993

Alliance Polygono-Trisetion Br.-Bl. et R. Tx. ex Marchall 1947

Association Astrantio-Trisetetum Knapp et Knapp ex Oberd. 1957
A.-T. typicum Knapp et Knapp 1952

A.-T. buphthalmetosum Knapp et Knapp 1952

A.-T. trollietosum Knapp et Knapp 1952

Order Arrhenatheretalia R. Tx. 1931

Alliance Arrhenatherion Koch 1926

Association Pastinaco-Arrhenatheretum Passarge 1964

P.-A. typicum Passarge 1964

$P$. A. medicagetosum lupulinae Passarge 1964

P.-A. lolietosum subass. nova

\section{DESCRIPTION OF THE ASSOCIATIONS}

Astrantio-Trisetetum Knapp et Knapp ex Oberd. 1957 (Table 1, rel. 1-44)

With most samples collected between $600 \mathrm{~m}$ and $1200 \mathrm{~m}$ a.s.l., this vegetation type is distributed up to approximately $1500 \mathrm{~m}$ a.s.l. Following the occurrence of plant species with core distribution areas in adjoining montane perennial herbaceous vegetation zones (e.g. Mulgedio-Aconitetea), in comparison to the Pastinaco-Arrhenatheretum association (mean $=35 \pm 6.2$ species per relevé), Astrantio-Trisetetum grasslands harbour a higher plant species diversity (mean $=52 \pm 7.5$ species per relevé). Furthermore, with 238 recorded species they represent one of the most diverse plant communities in Slovenia. Diagnostic species (differentiating and constant species) of the association are as follows: Astrantia major, Trisetum flavescens, Dactylis glomerata, Heracleum sphondylium, Festuca pratensis, Trollius europaeus, Tragopogon pratensis subsp. orientalis, Veronica chamaedrys, Crepis biennis, Hypericum maculatum, Veratrum album, Anthoxanthum odoratum, Briza media, Buphthalmum salicifolium, Carex montana, Carlina acaulis, Lotus corniculatus Pimpinella saxifrage, Plantago lanceolata, Polygala vulgaris, Potentilla erecta, Trifolium pratense, Anthyllis vulneraria, Bromopsis erecta, Centaurea jacea ssp. jacea, Festuca rubra, Leontodon hispidus, Leucanthemum ircutianum, Linum catharticum, Primula veris, Thymus pulegioides, Trifolium montanum, Cirsium oleraceum, Luzula campestris, Trifolium alpestre, Campanula scheuchzeri, Galium album, Stellaria graminea, Bellis perennis, Knautia drymeia, Vicia cracca, Rhinanthus freynii and Salvia pratensis (Knapp \& Knapp 1952; Machold 1991). The more extensive management of grasslands at 
higher altitudes appears to be the main reason for the presence of rather large numbers of the Festuco-Brometea dry grassland species, which we have found in this vegetation type. The most abundant were Anthyllis vulneraria, Briza media, Bromopsis erecta, Rhinanthus freynii, Pimpinella saxifraga and Salvia pratensis. The intergradion of different grassland types, in particular the $A s$ trantio-Trisetetum and Pastinaco-Arrhenatheretum association, causes an intermixing among species of both vegetation types, and problematizes of their syntaxonomy, i.e. in distinguishing the associations from each other. Within Astrantio-Trisetetum, three subassociations can be distinguished (Table 1): (1) Astrantio-Trisetetum typicum (relevés 1-16, Table 1) was found in the lower to upper montane belt (Lom pod Storžičem, Koprivnik, Topla, Selce near Vrsno), mainly on slopes exposed to the south and west. It is characterised by the permanent presence of Astrantia major. Other species that were present in higher frequencies in these samples are: Potentilla erecta, Cruciata glabra, Trifolium alpestre, Rhinanthus freynii and Luzula campestris. (2) Astrantio-Trisetetum buphthalmetosum (relevés 17-40, Table 1) harbours the most diverse vegetation samples. This subassociation was found at higher altitudes (on average $914 \mathrm{~m}$ ) and on slopes of higher inclination with more permanent water-flow and less intensive management. Consequently, species characteristic of dry grasslands, like Buphthalmum salicifolium, Gymnadenia conopsea, Galium verum ssp. verum, Briza media, Bromopsis erecta, Pimpinella saxifraga, Allium carinatum, and Dianthus hyssopifolius were found in higher frequencies in these samples. Ecologically and floristically the subassociation represents the transition to the Brometalia erecti order. (3) Astrantio-Trisetetum trollietosum (relevés 41-44, Table 1) was found only on more humid soils. Therefore, the absence of Brometalia-species is characteristic of this subassociation. The regular presence of species from wet meadows, like Veratrum album, Lychnis flos-cuculi and Trollius europaeus, indicates the transition to the alliance Molinion.

\section{Pastinaco-Arrhenatheretum Passarge 1964 (Table 2, rel. 1-47)}

In comparison to Astrantio-Trisetetum grasslands, the current distribution of the Pastinaco-Arrhe- natheretum association appears to depend more on land-use patterns and grassland management. The grasslands of this association are not restricted to a particular phytogeographical region. In Slovenia the association is widespread in the prealpine phytogeographical region. Along the edges of the alpine phytogeographical region it is often replaced by Astrantio-Trisetetum, while in the Dinaric region, where the management of grasslands is usually more extensive, transitions towards the dry grasslands of order Brometalia erecti could be followed (Škornik 2001). Characteristic species of the Pastinaco-Arrhenatheretum association are Pastinaca sativa and Campanula patula (Steinbuch 1995). In addition, other mesophilous species, like Achillea millefolium, Bellis perennis, Centaurea jacea subsp. jacea, Dactylis glomerata, Festuca pratensis, Knautia drymeia, Lathyrus pratensis, Leontodon hispidus, Leucanthemum ircutianum, Lotus corniculatus, Plantago lanceolata, Prunella vulgaris, Tragopogon pratensis subsp. orientalis, Trifolium medium, Trifolium pratense, Trisetum flavescens and Vicia cracca are well represented, with frequencies $>50 \%$. In more eutrophic stands species of Cynosurion grasslands, like Lolium perenne, Erigeron annuus, Trifolium repens and Veronica chamaedrys are common.

Within Pastinaco-Arrhenatheretum we identified the following three subassociations: (1) Pastinaco-Arrhenatheretum typicum (relevés 1-25, Table 2) is mainly distributed across the submontane belt, mostly on flat terrain, where more moderate intensive management practises of grassland are common. Typical species are Arrhenatherum elatius, Holcus lanatus, Ranunculus acris subsp. acris, Stellaria graminea and Heracleum sphondylium. (2) Pastinaco-Arrhenatheretum medicagetosum lupulinae shows thermophilic characteristics, and with higher abundance of Brometalia-species, like Briza media, Salvia pratensis, Anthoxanthum odoratum, Pimpinella saxifrage, Knautia arvensis, Medicago lupulina, Bromopsis erecta, and Galium verum ssp. verum, it represents the transition to the Brometalia erecti order (relevés 26-37, Table 2); (3) Pastinaco-Arrhenatheretum lolietosum subass. nova (relevés 38-47, Table 2, holotypus hoc loco: Table 2/29), which represents floristically poorer stands (probably owing to a more intensive management regime) with a higher proportion of species characteristic of the Lolio-Cynosuretum association such as Capsella bursa-pastoris, Lolium perenne and Phleum pratense. 


\section{Chorological spectrum AND Life} FORMS

Most vegetation samples were taken in the central and northern parts of Slovenia; the presence of European, Euroasiatic and Eurosiberian geoelements in high proportions was thus expected (Figure 2). Indeed, approximately half of all species found during vegetation sampling belongs to these groups. In the Astrantio-Trisetetum association Mediterranean-montane species were also regularly found, while species of Illyrian, SouthEastern European or Pontic origin were present only at low frequencies < 2\% (Figure 2). Owing to more intensive management cosmopolitan and adventive plants were more abundant in the Pastinaco-Arrhenatheretum association.

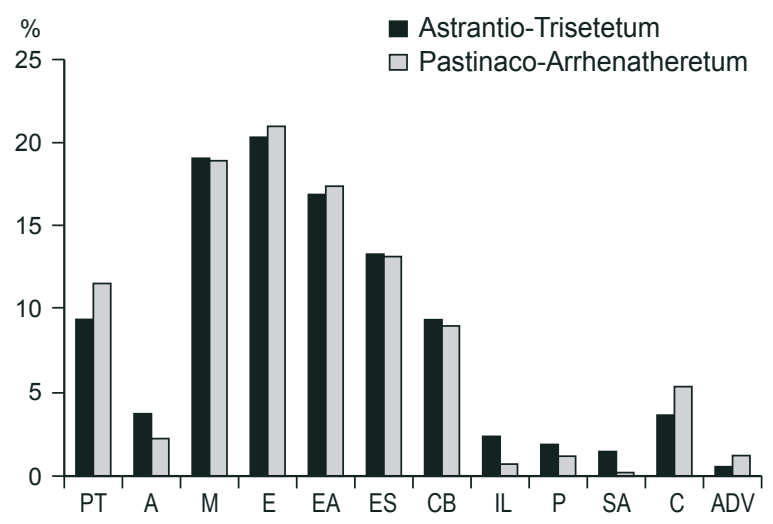

Figure 2: Chorological groups of the associations AstrantioTrisetetum and Pastinaco-Arrhenatheretum.

(Abbrevations: P - Paleotemperate, A - Alpine, M - Mediterranean, E - European, EA - Eurasian, ES - Eurosiberian, CB - Circumboreal, IL - Illyrian, P - Pontic, SA - Subatlantic, C - Cosmopolitans, ADV - Adventive plants).

Slika 2: Geoelementna sestava asociacij Astrantio-Trisetetum in Pastinaco-Arrhenatheretum.

(Okrajšave: P - paleotemperatne, A - alpinske, M - mediteranske, E - evropske, EA - evrazijske, ES - evrosibirske, $\mathrm{CB}$ - cirkumborealne, IL - ilirske, $\mathrm{P}$ - pontske, SA - subatlantske, C - kozmopoliti, ADV - adventivke).

Both associations are dominated by hemicryptophytes and thus represent the characteristic type of central European anthropogenic grassland (Figure 3). While we found more geophytes in stands of Astrantio-Trisetetum grasslands (11\%), therophytes are more abundant in Pastinaco-Arrhenatheretum association (ca. 10\%).

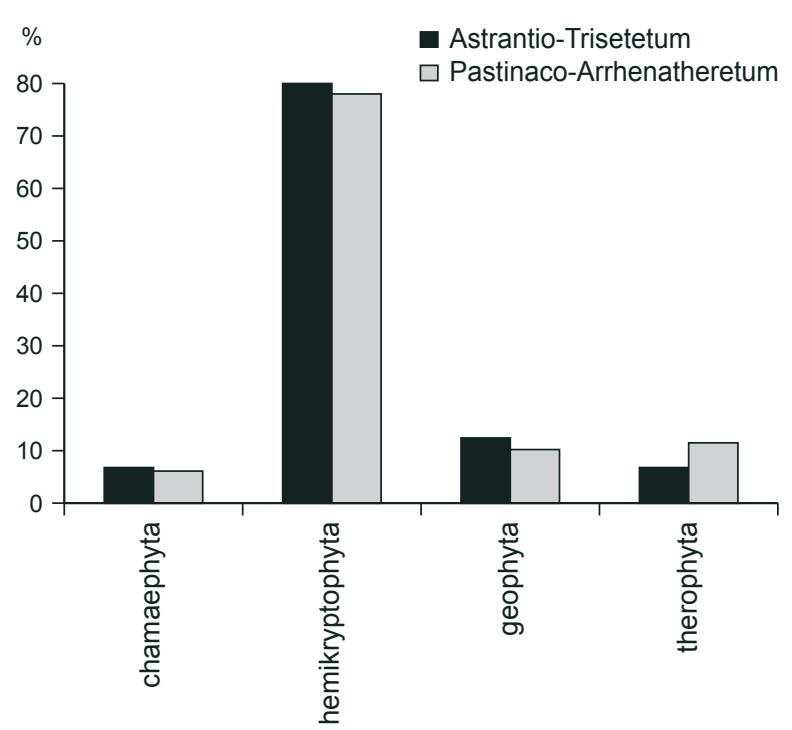

Figure 3: Specter of life forms of the associations AstrantioTrisetetum and Pastinaco-Arrhenatheretum.

Slika 3: Spekter življenjskih oblik v asociacijah AstrantioTrisetetum in Pastinaco-Arrhenatheretum.

\section{ECOLOGICAL CONDITIONS}

Figure 4 shows the effects of the abiotic variables on the species composition of these grasslands. In the RDA of all 91 vegetation relevés of the associations Astrantio-Trisetetum and PastinacoArrhenatheretum, relevé scores of axis 1 were positively correlated with altitude and pastures but negatively with meadows. The ordination biplot shows relevés of the Astrantio-Trisetetum association at the right side, which means that those stands are frequently associated with higher altitudes and are more often used as pasture than Pastinaco-Arrhenatheretum stands. On the other hand stands of the Pastinaco-Arrhenatheretum association are characteristically used as meadows and associated with higher air temperatures (lower altitudes) and humid, nutrient-rich soils. Therefore, many mesic species from lowland, intensive-use grasslands like Arrhenatheretum elatius, Ranunculus acris, Dactylis glomerata, Tragopogon pratensis, Daucus carota, Plantago lanceolata, Erigeron annuus, Pastinaca sativa and Trifolium medium were found with higher frequencies in Pastinaco-Arrhenatheretum meadows. The ordination biplot shows the species related to AstrantioTrisetetum relevés on the right side. Species, such as Nardus stricta, Potentilla erecta, Polygala vulgar- 


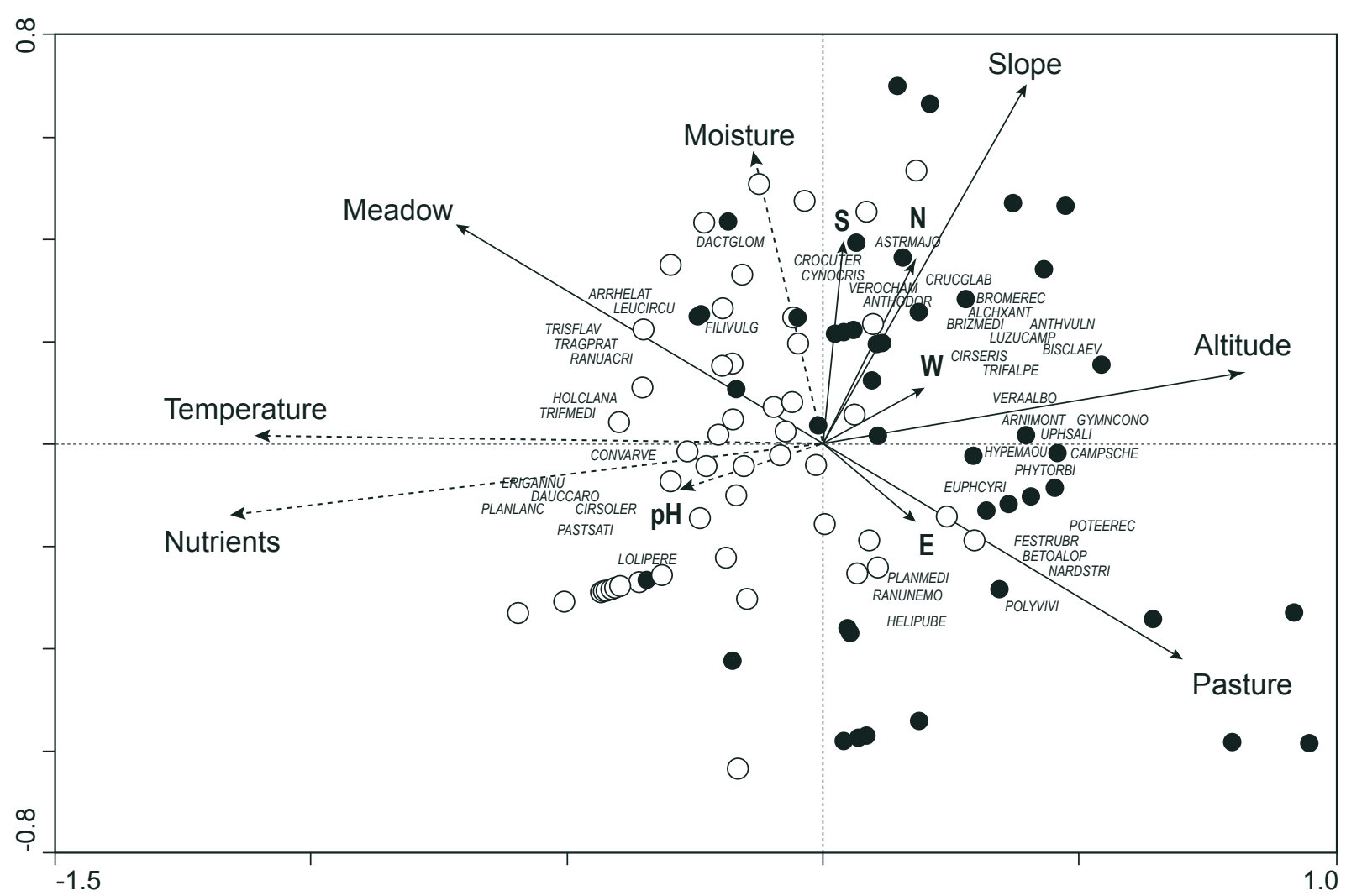

Figure 4: RDA ordination diagram of relevés ( $\mathrm{n}=91,108$ species) and environmental variables of studied Trisetum flavescens grasslands in Slovenia. Eigenvalues: RDA axis $1=0.125$; RDA axis $2=0.056$. Shown species have the highest weight. The mean Ellenberg indicator values for temperature, moisture, soil reaction $(\mathrm{pH})$ and nutrients were added as supplementary variables (dashed lines) without any effect on the analysis. $\bigcirc$ - relevés of Pastinaco-Arrhenatheretum; - relevés of Astrantio-Trisetetum. Abbrevations: S - south; $\mathrm{N}$ - nord; E - east; W - west; $\mathrm{pH}$ - soil reaction. Abbrevations of species are explained in Tables 1 and 2.

Slika 4: Ordinacijski diagram RDA-analize popisov in okoljskih spremenljivk obravnavanih travišč. Lastne vrednosti: RDA os $1=0.125$; RDA os $2=0.056$. Prikazane so vrste $\mathrm{z}$ največjo težo. Vrednosti Ellenbergovih indeksov za temperaturo, vlažnost rastišča, kemijsko reakcijo tal $(\mathrm{pH})$ in hranilnost tal so bile upoštevane kot pasivne spremenljivke in niso imele vpliva na rezultate analize. $\bigcirc$ - popisi asociacije Pastinaco-Arrhenatheretum; - popisi asociacije Astrantio-Trisetetum. Okrajšave: $\mathrm{S}$ - jug; N - sever; E - vzhod; W - zahod; pH - kemijska reakcija tal. Kratice vrst so obrazložene v tabelah 1 in 2.

is, Plantago media and Euphorbia cyparissias, which are common for less humid and nutrient-poor soils, are at the bottom right. At the top right side appear species frequent in extensively used pastures at higher altitudes, such as Biscutella laevigata, Phyteuma orbiculare, Arnica montana and Veratrum album.

\section{Conservation implication}

In contrast to other central European countries which have formerly been a focus for the occurrence of Arrhenatherion and Trisetion grasslands (Oberdorfer 1983), stands of both alliances are currently still widespread in Slovenia. However, the ploughing up of grassland into arable fields and the sowing of commercial grass mixtures currently constitute the major problems for the maintenance of Arrhenatherion and Trisetion grassland habitats in Slovenia.

According to Ellenberg (1996) the most appropriate management regime for typical $A r$ rhenatherum elatius grassland involves cutting twice per year and fertilization with cattle manure. More intensive management impairs the vitality and abundance of plant species, while the application of liquid manure causes an increase in Apiaceae species, like Anthriscus sylvestris and Heracleum sphondylium, which decrease the nutri- 
ent value of meadows (Ellmauer \& Mucina 1993). More intensively managed Arrhenatherion meadows are replaced even over a short time period by uniform, species-poor stands of the Cynosurion alliance (Oberdorfer 1983). Similar management regimes, with the application of cattle manure (each second year) and low cutting frequencies (1-2 cuttings per year), are also suggested for the maintenance of the Polygono-Trisetion grasslands being studied.

Besides an appropriate, low to moderate intensity management, for maintaining a representative portion of Slovenia's T. flavescens grassland habitats, it will be necessary to establish a network of appropriately-sized, protected grassland areas that are span a range of altitudes in both the submontane and montane altitudinal belts.

\section{CONCLUSIONS}

In the present study secondary grasslands dominated by Trisetum flavescens have been investigated throughout the submontane and montane regions of Slovenia.

According to the plant species composition and abundance of the plants these grasslands were classified into two associations from two different alliances, i.e. (1) Astrantio-Trisetetum (Polygono-Trisetion alliance) and (2) PastinacoArrhenatheretum (Arrhenatherion alliance). While stands of the Astrantio-Trisetetum association are found mainly at higher altitudes, stands of the Pastinaco-Arrhenatheretum association can be found at lower altitudes and on soils with higher nutrient content. Grasslands of the AstrantioTrisetetum association are more species rich, with species characteristic of primary grasslands above the tree line. The high species richness of this vegetation type could also be explained as a consequence of low-intensity management. A topography that restricts the use of farm machinery, a shorter vegetation period and greater distances to farming estates are the main reasons for less frequent mowing at higher altitudes (Ellmauer \& Mucina 1993). In contrast the dominant plant species of Pastinaco-Arrhenatheretum indicate more intensive management. Besides the more intensive management, the neighbourhood of eutrophic stands of the Cynosurion alliance is another factor that exerts an essential influence on the species composition and diversity of Pastinaco-Arrhenatheretum grasslands.

\section{POVZETEK}

Travišča s prevladujočim rumenkastim ovsencem (Trisetum flavescens) $\mathrm{v}$ submontanskih in montanskih predelih Slovenije

V članku so predstavljene fitocenološke in ekološke značilnosti travišč s prevladujočim rumenkastim ovsencem (Trisetum flavescens) v montanskih in submontanskih predelih Slovenije. Vegetacijski popisi so bili vzorčeni na traviščih $\mathrm{z}$ zmerno intenzivnim gospodarjenjem, večinoma $v$ letih 2005 in 2006, na nadmorski višini med $400 \mathrm{~m}$ in 1.500 $\mathrm{m}$. Popisovanje vegetacijskih sestojev je potekalo po standardni srednjeevropski Braun-Blanquetovi metodi. Na terenu je bilo popisanih 91 sestojev obravnavanih travišč. Sintaksonomska pripadnost zbranih popisov je bila ugotovljena na osnovi primerjave lastnih popisov z 98 referenčnimi popisi iz literature s pomočjo multivariatnih statističnih metod (TWINSPAN, PCA, RDA analiz). Pri posameznih popisih so zabeleženi: (1) lokacija, (2) nadmorska višina (m), (3) nebesna lega, (4) naklon pobočja $\left({ }^{\circ}\right)$, (5) geografske koordinate, (6) pedološke enote in (7) število vrst. Za popise je podana ocena ekoloških razmer na rastiščih s pomočjo fitoindikatorskih metod (Ellenberg in sod. 1991), pri čemer so bili Ellenbergovi indeksi določeni za naslednje parametre: (1) temperaturo; (2) vlažnost rastišča; (3) kemijsko reakcijo tal $(\mathrm{pH})$ in (4) hranilnost tal. Za določitev vpliva izbranih merjenih in ocenjenih okoljskih spremenljivk na floristično sestavo proučevanih travišč je bila uporabljena redundančna (RDA) analiza. Na osnovi statističnih analiz, floristične zgradbe in abundance vrst so bili obravnavni travniki in pašniki uvrščeni v dve asociaciji, in sicer v asociacijo Astrantio-Trisetetum (zveza Polygono-Trisetion) ter v asociacijo Pastinaco-Arrhenatheretum (zveza Arrhenatherion). Nadaljnja členitev na nižje sintaksonomske enote je pogojena predvsem $\mathrm{z}$ različno stopnjo intenzivnosti gospodarjenja $\mathrm{s}$ travišči, $\mathrm{z}$ edafskimi razmerami in nadmorsko všino. Tako so bile v asociaciji Astrantio-Trisetetum opisane naslednje nižje sintaksonomske skupine: (1) subasociacija Astrantio-Triseteum typicum, ki v sinekološkem smislu predstavlja prehode med redovoma Brometalia erecti in Molinietalia, (2) subasociacija Astrantio-Triseteum buphthalmetosum, ki je od vseh subasociacij vrstno najbogatejša in v ekološkem in florističnem pogledu predstavlja prehod med redom Brometalia erecti in osrednjo obliko asociacije Astrantio-Trisetetum, in (3) subasociacija Astrantio-Triseteum trollietosum, v kateri se pojavljajo značilnice vlažnih travišč. Asociacijo Pastinaco- 
Arrhenatheretum delimo na naslednje nižje sintaksonomske enote: (1) subasociacijo Pastinaco-Arrhenatheretum typicum, za katero je značilna zmerna gospodarska raba travišč, (2) termofilno subasociacijo Pastinaco-Arrhenatheretum medicagetosum lupulinae $\mathrm{z}$ večjo prisotnostjo vrst suhih travišč in (3) subasociacijo Pastinaco-Arrhenatheretum lolietosum subass. nova, kjer je vnos hranilnih snovi večji.

Rezultati redundančne analize, narejeni na osnovi floristične sestave ter merjenih in ocenjenih okoljskih dejavnikov, so pokazali, da je vrstna sestava obravnavanih travišč v najmočnejši povezavi $\mathrm{z}$ nadmorsko višino in načinom gospodarjenja (paša ali košnja). Travišča asociacije Astrantio-Trisetetum so tako predvsem pašniki, ki se pojavljajo na višjih nadmorskih višinah, sestoji asociacije $P a$ stinaco-Arrhenatheretum pa košeni travniki na nižjih nadmorskih višinah, floristična sestava pa nakazuje s hranili bogata tla. V sestojih obeh asociacij prevladujejo evropske, evrazijske in evrosibirske vrste, v asociaciji Astrantio-Trisetetum pa se pogosteje pojavljajo tudi mediteransko-montanske vrste; kozmopolitov in adventivk pa je več v asociaciji Pastinaco-Arrhenatheretum. Od življenjskih oblik v obeh asociacijah prevladujejo hemikriptofiti. V asociaciji Astrantio-Trisetetum so razmeroma pogosti še geofiti, v asociaciji Pastinaco-Arrhenatheretum pa terofiti. Travišča zvez Polygono-Trisetion in Arrhenatherion so v Sloveniji še razmeroma pogosta, vendar se zaradi povečane intenzivne rabe, njihove površine na številnih območjih spreminjajo $\mathrm{v}$ vrstno revnejše sestoje ljulke ali travniškega lisičjega repa. Z vidika biotske raznovrstnosti je problematično tudi izginjanje travišč zaradi povečanega obsega obdelovalnih površin, zaradi sejanja travnih mešanic in fragmentacije teh travišč. Za ohranjanje diverzitete vrst ter strukture in funkcije obravnavanih travišč bi bilo potrebno primerno upravljanje - zmerno gnojenje in košnja enkrat ali dvakrat na leto oziroma kombiniranje košnje in paše. Določena travišča na večjih površinah, ki so dovolj reprezentativna s karakterističnimi vrstami, pa bi bilo potrebno zajeti v ustrezni varstveni režim in omogočiti disperzijo njihovih vrst (z migracijskimi koridorji oz. s transhumanco).

\section{ACKNOWLEDGEMENTS}

The research was carried out in the research programme "Biodiversity" "(contract number P10078) headed by B. KRYŠTUFEK, and founded by the Slovenian Research Agency.
I. DAKSKOBLER and two anonymous authors have made a significant contribution to revision of the article.

\section{REFERENECES}

Aichinger, E. 1933: Vegetationskunde der Karavanken. Pflanzensociologie, 2, Jena, G. Fischer.

Atkinson, P.W., Buckingham, D. \& Morris, A. J. 2004: What factors determine where invertebrate-feeding birds forage in dry agricultural grasslands? Ibis 146: 99-107.

Čarni, A. 2001: Vegetation of Cultivated grasslands in the Goričko region (NE Slovenia). Acta Biologica Slovenica 44 (4) 13-27.

Dierschke, H. 1994: Pflanzensociologie. Grundlagen und methoden. Ulmer, Sttutgart.

Dierschke, H. 2002: Kulturgrasland. Wiesen, Weiden und verwandte Staudenflora. Stuttgart, E. Ulmer.

Ellenberg, H. 1996: Vegetation Mitteleuropas mit den Alpen in ökologischer, dynamischer und historischer Sicht. 5. Aufl. Eugen Ulmer Verlag, Stuttgart.

Ellenberg, H., Weber, H.E., Düll, R., Wirth, V. \& Werner, W. 2001: Zeigerwerte von Pflanzen in Mitteleuropa. Scripta Geobotanica 18, Göttingen.

Ellmauer, T. \& Mucina, L. 1993: Molinio-Arrhentheretea. In: Mucina, L., Grabherr G. \& Ellmauer, T. (eds.): Die Pflanzengesellschaften Österreichs. Teil I. Anthropogene Vegetation. Gustav Fischer Verlag Jena, Stuttgart: 297-401.

Gomez-Pompa, A. \& Kaus, A. 1992: Taming the wilderness myth. Bioscience 42: 271-279.

Hill, M.O. \& Gauch, H.G. 1980: Detrended correspondence analysis: an improved technique. Vegetatio 42:47-58.

Hill, M.O. \& Šmilauer, P. 2005: TWINSPAN for Windows version 2.3. Centre for Ecology and Hydrology \& University of South Bohemia, Huntingdon \& České Budějovice.

Horvatić, S., Tomažič, G. 1941: Travniška vegetacija reda Arrhenatheretalia v nižinskem pasu Slovenije. Zbornik prirodoslovnega društva, 2: $68-75$.

Jongman, R.H.G., ter Braak C. J. F. \& van Tongeren O.F.R. 1995: Data analysis in community and landscape ecology. Cambridge University Press, Cambridge.

Kladnik, D. \& Gabrovec, M. 1998: Raba tal. In: 
Fridl, J. et al. (eds.): Geografski atlas Slovenije. DZS, Ljubljana.

Kladnik, D. 1998: Alpski svet. In: Perko, D. \& Orožen Adamič, M. (eds.): Slovenija, pokrajine in ljudje. Založba Mladinska knjiga, Ljubljana, pp. 34-53.

Kladnik, D. 1998a: Dinarski svet. In: Perko, D. \& Orožen Adamič, M. (eds.): Slovenija, pokrajine in ljudje. Založba Mladinska knjiga, Ljubljana, pp. 296-311.

Knapp, G., Knapp, R. 1952: Über die Goldhafer-Wiesen (Trisetetum flavescentis) im nördlichen Vorarlberg und im Obrallgäu. Landwirt. Jahrb. Bayern, 29: 239-256.

Machold, C. 1991: Die Trespenwiesen des Walgaus. Diplomarbeit, Univ. Wien.

Marinček, L. 1987: Bukovi gozdovi na Slovenskem. Delavska enotnost, Ljubljana.

Martinčič, A., Wraber, T., Jogan, N., Ravnik, V., Podobnik, A., Turk, B. \& Vreš, B. 2007: Mala flora Slovenije. Ključ za določanje praprotnic in semenk. Tehniška založba Slovenije, Ljubljana.

McCracken, D.I. \& Tallowin, J.R. 2004: Swards and structure: the interection between farming practices and bird food resources in lowland grasslands. Ibis 146: 108-114.

McKinney, M.L. \& Lockwood, J.L. 1999: Biotic homogenisation: a few winners replacing many losers in the next mass extinction. Trends in Ecology and Evolution 14: 450-453.

Oberforster, M. 1986: Beitrag zur Kenntnis der Böden und vegetation von Futterwisen, Weiden und Feuchtbeständen im Oberösterreichischen Voralpengebiet (Untersuchungen in den Gemeinden Grossraming und Maria Neustift). Diplomarbeit, Univ. f. Bodenkultur, Wien.

Oberdorfer, E. 1983: Klasse: Molinio-Arrhenatheretea Tx. 37 (em. Tx. et Prsg. 51). In: Oberdorfer, E. (ed.), Süddeutsche Pflanzengesellschaften. Teil III. 2. Aufl. pp. 346-436. Gustav Fischer Verlag, Jena.

Pärtel, M., Bruun, H.H. \& Sammul, M. 2006: Biodiversity in temperate European grasslands: origin and conservation. Grassland Science in Europe 10:14.

Poldini, L \& Oriolo, G. 1994: La vegetatione dei prati da sfalcio e dei pascoli intensive (Arrhenatheretalia e Poo-Trisetetalia) in Fruli (NE Italia). Studia Geobotanica 14(1): 3-48.

Poldini, L. 2001: Atlante corologico delle piante vascolari nel Friuli - Venezia Giulia. Inventario floristico regionale. Unversita degli studi di Trieste dipartemento di biologia, Udine.
Pott, R. 1995: Die Pflanzengesellschaften Deutschlands. Verlag Eugen Ulmer Stuttgart.

Seliškar, A. 1993: Übersicht der Wiesenvegetation der Kamniško-Savinjske Alpen. In: Zupančič, M. (ed.): Flora in vegetacija Kamniško-Savinjskih Alpe. Flora und Vegetation der Kamniško-Savinjske Alpen. Referati. Biološki inštitut ZRC SAZU, Ljubljana.

Steinbuch, E. 1995: Wiesen und Weiden der Ost-, Süd- und Weststeiermark. Dissertationes Botanica. Gebrüder Borntraeger Verlagsbuchhandlung, Berlin, Stuttgart.

Swift M.J., Vandermeer, P.S., Ramakrishnan, J.M., Anderson, C.K. Ong \& Hawkins, B.A. 1996: Biodiversity and Agroecosystem Function. In: Mooney, H. A. (ed.): Functional roles of biodiversity: a global perspective. Chichester, J. Wiley \& sons.

Škornik, S. 2000: Suha in polsuha travišča reda Brometalia erecti Koch 1926 v Sloveniji. Doktorska disertacija. Univerza v Ljubljani. Biotehniška fakulteta. Oddelek za biologijo.

Ter Braak, C.J.F. \& Šmilauer, P. 2002: CANOCO reference manual and CnoDraw for Windows user's guide. Software for Cannonical Community Ordination (version 4.5). Microcomputer Power, Ithaca, New York, USA.

Walker, K.J., Stevens, P.A., Stevens, D.P., Mountford, J.O., Manchester, S.J. \& Pywell, R.F. 2004: The restoration and re-creation of species-rich lowland grassland on land formerly managed for intensive agriculture in the UK. Biological Conservation 119: 1-18.

Westhoff, V. \& van der Maarel, E. 1973: Ordination and classification of communities. Handbook of Vegetation Science 5: 617-737, Junk. The Hague.

Wilson, J.D., Evans, A.D. \& Grice, P.V. 2009: Bird Conservation and Agriculture. Cambridge University Press, Cambridge UK, 394 pp.

Žiberna, I. 1998: Strojna, Kozjak in Pohorje. In: Perko, D., Orožen Adamič, M. (ed.): Slovenija, pokrajine in ljudje. Založba Mladinska knjiga, Ljubljana, pp. 142-155. 


\section{APPENDIX}

Appendix to the table 1: Localities, dates of the relevés and species, occurring in one relevé only. Dodatek k tabeli 1: Lokalitete popisanih sestojev, datumi popisov ter vrste, ki se pojavljajo samo v enem popisu.

1: Koprivnik (Bohinj), 15.7.2005, Phleum phleoides, Vaccaria pyramidata; 2: Brčev rovt (Hrasti), 17.7.2005, Peucedanum austriacum, P. cervaria; 3: Brčev rovt (Hrasti), 3.7.2006; 4: Lom pod Storžičem, 4.7.2006; 5: Jelendol, 6.8.2006; 6: Završnik, 8.8.2006; 7: pod Storžičem, 4.7.2006; 8: pod Kofcami, 5.7.2006; 9: Završnik, 8.8.2006; 10: Grahovše, 10.7.2005, Campanula rotundifolia; 11: Jezersko, 19.7.2006; 12: Koprivnik (Bohinj), 26.7.2006; 13: Cirkuše (Tuhinjska dolina), 7.7.2006; 14: Topla, 24.6.2006, Arabidopsis thaliana; 15: Selce pod Vrsnim, 22.7.2005; 16: Lom pod Storžičem, 4.7.2006; 17: Potarje, 10.7.2005, Trifolium aureum; 18: Brčev rovt (Hrasti), 17.7.2005, Lathyrus tuberosus, Polygonatum vericillatum, Vaccinium vitis-idaea; 19: Gozd, 18.7.2005, Helleborus odorus; 20: Brčev rovt (Hrasti), 3.7.2006, Polygala comosa; 21: pod Kriško goro, 24.7.2006, Aconitum vulparia, Scabiosa lucida; 22: Robanova planina, 7.7.2005, Salvia verticillata; 23: Bohinj (Kranjska dolina), 15.7.2005, Brachypodium rupestre, Luzula sylvatica, Thesium linophyllon, T. pyrenaicum; 24: Pikovo, 22.6.2005; 25: pod Kofcami, 5.7.2006; 26: Podpeca, 22.6.2005, Alchemilla glaucescens; 27: Zg. Jezersko, 18.7.2005; 28: Zg. Jezersko, 18.7.2005; 29: Korensko sedlo, 20.7.2005; 30: pod Kofcami, 5.7.2006; 31: Gozd, 24.7.2006; 32: Prevala, 14.7.2005, Carduus crispus, Carex nigra, Knautia longifolia, Orchis ustulata, Plantago major, Stachys alpina, Soldanella alpina, Verbascum nigrum; 33: Vršič-Trenta, 21.7.2005, Aster amellus, Carduus crassifolius ssp. glaucus, C. nutans, Dianthus barbatus, Epipactis atrorubens, Erigeron acris, Scorzonera rosea; 34: Trenta, 21.7.2005; 35: Trenta, 21.7.2005; 36: Marija Snežna (Breginj), 22.7.2005, Peucedanum schottii, Rumex alpestris, Sedum maximum; 37: Lepena, 22.7.2005; 38: Radovna, 20.7.2005, Ajuga genevensis, Cirsium arvense, Lythrum salicaria, Melittis melissophyllum; 39: Vrsno, 22.7.2005, Convolvulus arvensis; 40: Pikovo, 22.6.2005, Medicago falcata, Selinium carvifolia; 41: pod Storžičem, 4.7.2006, Cirsium rivulare, Vicia sylvatica; 42: Zatrnik, 26.7.2006; 43: Lom pod Storžičem, 4.7.2006; 44: Robanov kot, 25.6.2006, Veronica officinalis.
Appendix to the table 2: Localities, dates of the relevés and species, occurring in one relevé only. Dodatek k tabeli 2: Lokalitete popisanih sestojev, datumi popisov ter vrste, ki se pojavljajo samo v enem popisu.

1: Podvoljek, 25.6.2006; 2: Šober (Kozjak), 1.7.2006; 3: Sopotnica pri Škofji Loki, 6.7.2006; 4: Modrič (Pohorje), 23.6.2006, Centaurea cyanus; 5: Malo Tinje, 23.6.2006; 6: Lenart pri Gornjem Gradu, 25.6.2006, Campanula rapunculoides; 7: Podolševa, 24.6.2006, Cardaminopsis halleri; 8: Podlom (pod Podvoljekom), 25.6.2006; 9: Malo Tinje, 23.6.2006; 10: Pesnik (Pohorje), 23.6.2006, Carex montana, Viola tricolor; 11: Topla, 24.6.2006; 12: Planina pod Šumikom, 23.6.2006; 13: Bohinjska Bela, 26.7.2006, Medicago falcata; 14: Obrne, 26.7.2006; 15: Selo pri Bledu, 31.7.2006; 16: Hlebce pri Lescah, 31.7.2006; 17: Jelendol, 6.8.2006; 18: Cimper (Tržič), 8.8.2006, Astrantia major; 19: Vetrno, 18.7.2005, Melittis mellisophyllum; 20: Veliko Tinje, 23.6.2006; 21: Topla, 24.6.2006, Melica nutans; 22: Podvoljek, 25.6.2006; 23: Tirosek (pred Šmiklavžem), 25.6.2006, Ononis arvensis; 24: Maček pod Šobrom, 16.6.2006, Carex brizoides, Vicia dumetorum; 25: Kebelj, 23.6.2006; 26: Četna ravan, 6.7.2006, Carlina vulgaris; 27: Tuhinjska dolina, 9.6.2006, Chamaecytisus hirsutus, Inula salicina; 28: Stara Fužina, 15.7.2006; 29: Slatna (Begunje), 31.7.2006, Thalictrum aquilegiifolium; 30: Grčarice, 29.7.2007, Verbascum austriacum, Calamagrostis vilosa, Reseda lutea; 31: Retje, 30.7.2007, Aquilegia atrata, Cirsium pannonicum, Inula hirta, Lilium bulbiferum, Rhinanthus minor, Scabiosa columbaria; 32: Breginj, 22.7.2005, Carex divulsa, Erigeron acris, Fragaria vesca, Geranium phaeum, Teucrium chamaedrys; 33: Breznica pod Lubnikom, 6.7.2006; 34: Šober (Kozjak), 1.7.2006, Carex fritschii; 35: Kozjak (kmetija Sluga), 1.7.2006, Dianthus armeria; 36: Rdeči breg (pod Kamenikom), 1.7.2006, Reseda luteola; 37: Florjan pri Gornjem Gradu, 25.6.2006, Lathyrus sylvestris; 38: Cerkno, 6.7.2006; 39: Zvirče, 31.7.2006; 40: Zg. Fužine, 18.7.2005, Cichorium intybus, Lolium temulentum; 41: Čeplez (Cerkno), 6.7.2006, Acinos alpinus; 42: proti Bašlju, 24.7.2006; 43: Sr. vas v Bohinju, 15.7.2005; 44: Jarčje brdo, 20.7.2005, Fragaria viridis, Gentiana asclepiadea, Lathyrus hirsutus, Lysimachia vulgaris; 45: Dolenčice, 20.7.2005, Dianthus barbatus; 46: Bašelj, 10.7.2005, Agrimonia eupatoria, Plantago major, Polygonum aviculare, Ranunculus repens; 47: Povje, 10.7.2005, Cerastium arvense, Pedicularis verticillata. 
Table 1: Analytical table of the association Astrantio-Trisetetum Knapp et Knapp ex Oberd. 1957.

Tabela 1: Analitična tabela asociacije Astrantio-Trisetetum Knapp et Knapp ex Oberd. 1957.

\begin{tabular}{|c|c|c|c|c|c|c|c|c|c|c|c|c|c|c|c|c|}
\hline Number of relevé (Številka popisa) & 1 & 2 & 3 & 4 & 5 & 6 & 7 & 8 & 9 & 10 & 11 & 12 & 13 & 14 & 15 & 16 \\
\hline Altitude (m) (Nadmorska višina) & 950 & 620 & 615 & 680 & 860 & 900 & 860 & 975 & 880 & 860 & 900 & 1005 & 600 & 1180 & 420 & 625 \\
\hline Releve area (m) (Velikost popisne ploskve) & 50 & 25 & 25 & 50 & 50 & 50 & 25 & 25 & 50 & 50 & 25 & 25 & 25 & 25 & 25 & 25 \\
\hline Exposition (Lega) & NE & S & S & $\mathrm{N}$ & NE & SW & NW & SW & $\mathrm{W}$ & SW & $\mathrm{W}$ & $\mathrm{W}$ & $\mathrm{S}$ & $\mathrm{S}$ & 0 & E \\
\hline Inclination $\left(^{\circ}\right)$ (Naklon pobočja) & 5 & 20 & 20 & 5 & 35 & 5 & 20 & 30 & 30 & 20 & 10 & 30 & 35 & 30 & 0 & 5 \\
\hline $\begin{array}{l}\text { X-coordinate } \\
\text { (X-koordinata) }\end{array}$ & 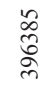 & $\begin{array}{l}\frac{n}{2} \\
\hat{n} \\
\tilde{m} \\
n\end{array}$ & 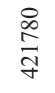 & $\begin{array}{l}m \\
\infty \\
\infty \\
\tilde{m}\end{array}$ & $\begin{array}{l}\overrightarrow{ } \\
\infty \\
\infty \\
\dot{f}\end{array}$ & 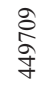 & 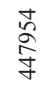 & 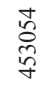 & $\begin{array}{l}8 \\
: \\
\circ \\
\dot{f}\end{array}$ & $\begin{array}{l}\text { त̂ } \\
\stackrel{\infty}{\infty} \\
\text { वे }\end{array}$ & $\begin{array}{l}\text { fo } \\
\text { 足 } \\
\text { f }\end{array}$ & $\begin{array}{l}\text { f } \\
\text { के } \\
\text { J }\end{array}$ & $\frac{\stackrel{0}{+}}{\stackrel{+}{f}}$ & $\begin{array}{l}\overrightarrow{8} \\
\stackrel{8}{f}\end{array}$ & 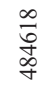 & 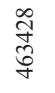 \\
\hline $\begin{array}{l}\text { Y-coordinate } \\
\text { (Y-koordinata) }\end{array}$ & $\begin{array}{l}\text { ず } \\
\text { g }\end{array}$ & $\begin{array}{l}\text { त్ర } \\
\text { ¿্ড }\end{array}$ & 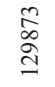 & $\begin{array}{l}\text { } \\
\text { }\end{array}$ & $\begin{array}{l}\infty \\
\text { ले } \\
\text { }\end{array}$ & $\begin{array}{l}\stackrel{\partial}{+} \\
\text { mे }\end{array}$ & $\begin{array}{l}\text { ఫेँ } \\
\text { ले } \\
\text { }\end{array}$ & $\frac{⿱ 亠 䒑}{\stackrel{n}{n}}$ & 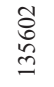 & 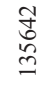 & 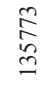 & 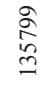 & $\underset{\stackrel{n}{\infty}}{\stackrel{m}{n}}$ & $\underset{\substack{\infty\\
}}{\stackrel{\infty}{n}}$ & 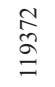 & $\begin{array}{l}\infty \\
\stackrel{2}{o} \\
\stackrel{8}{+} \\
\end{array}$ \\
\hline $\begin{array}{l}\text { Pedologic cartographic unit } \\
\text { (Pedološka kartografska enota - FAO) }\end{array}$ & $\sum_{j}^{0}$ & $\sum_{j}^{\bar{J}}$ & $\stackrel{3}{\vec{\mu}}$ & છ & $\sum_{0}^{\infty}$ & $\frac{\ddot{a}}{\vec{a}}$ & $\frac{\ddot{Z}}{\vec{a}}$ & 宵 & $\sum_{j}^{\infty}$ & $\sum_{U}^{\infty}$ & $\sum_{0}^{\infty}$ & ઘ & છ & $\sum_{j}^{0}$ & છ & $\sum_{0}^{0}$ \\
\hline $\begin{array}{l}\text { Pedologic cartographic unit } \\
\text { (Pedološka kartografska enota - PKE) }\end{array}$ & $\stackrel{\vec{n}}{=}$ & $\stackrel{\circ}{0}$ & $\hat{\widehat{N}}$ & $\stackrel{\not}{\stackrel{0}{O}}$ & 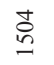 & $\stackrel{\curvearrowleft}{\cong}$ & $\stackrel{\cong}{\cong}$ & ڤั) & 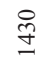 & $\stackrel{\check{\vartheta}}{\Xi}$ & $\stackrel{\check{\vartheta}}{\Xi}$ & $\stackrel{8}{\circ}$ & $\stackrel{\infty}{=}$ & 品 & $\stackrel{\infty}{=}$ & $\underset{\Xi}{\Xi}$ \\
\hline Cover (\%) (Pokrovnost) & 100 & 100 & 100 & 100 & 100 & 100 & 100 & 100 & 100 & 100 & 100 & 100 & 100 & 100 & 100 & 100 \\
\hline Number of species (Število vrst) & 49 & 64 & 55 & 55 & 53 & 53 & 53 & 51 & 53 & 49 & 47 & 54 & 44 & 40 & 47 & 52 \\
\hline
\end{tabular}

Characteristic and differentiating species of the association

Listera ovata LISTOVAT

Carex montana CAREMONT

Characteristic and differentiating species of the subassociation typicum Knapp et Knapp ex Oberd. 1957

\begin{tabular}{|c|c|c|c|c|c|c|c|c|c|c|c|c|c|c|c|c|c|}
\hline Potentilla erecta & POTEEREC & 2 & + & + & 1 & + & 2 & . & + & + & 1 & + & + & + & . & + & 1 \\
\hline Luzula campestris & LUZUCAMP & . & + & + & + & + & . & + & + & . & + & + & + & + & + & . & + \\
\hline Trifolium alpestre & TRIFALPE & + & + & + & + & + & + & + & + & + & . & + & . & + & . & + & 1 \\
\hline Astrantia major & ASTRMAJO & + & 4 & 3 & 3 & 3 & 2 & 2 & 4 & 2 & 4 & 2 & 2 & 2 & 1 & . & 3 \\
\hline
\end{tabular}

Characteristic and differentiating species of the subassociation buphthalmetosum Knapp et Knapp ex Oberd. 1957

Briza media

BRIZMEDI

Bromopsis erecta

Buphthalmum salicifolium

BROMEREC

BUPHSALI

Galium verum ssp. verum

GALIVERU

Pimpinella saxifraga

PIMPSAXI

Gymnadenia conopsea

GYMNCONO

Allium carinatum

ALLICARI

Dianthus hyssopifolius

DIANHYSS

Characteristic and differentiating species of the subassociation trollietosum Knapp et Knapp ex Oberd. 1957

Cirsium oleraceum

Veratrum album

Lychnis flos-cuculi

CIRSOLER

Trollius europaeus

VERAALBU

LYCHFLCU

TROLEURO

Polygono-Trisetion Br.-Bl. et R. Tx. ex Marschall 1947 nom. inv.

Crocus vernus

CROCVERN

Geranium sylvaticum

GERASYLV

Poo alpinae-Trisetetalia Ellmauer et Mucina 1993

Campanula scheuchzeri

CAMPSCHE

Ranunculus nemorosus

RANUNEMO

Poa alpina

POAALPI

Pastinacio-Arrhenatheretum Passarge 1964

Arrhenatherum elatius

Campanula patula

ARRHELAT

CAMPPATU 
\begin{tabular}{cccccccccccccccccccccccccccc}
17 & 18 & 19 & 20 & 21 & 22 & 23 & 24 & 25 & 26 & 27 & 28 & 29 & 30 & 31 & 32 & 33 & 34 & 35 & 36 & 37 & 38 & 39 & 40 & 41 & 42 & 43 & 44 \\
\hline 875 & 620 & 910 & 615 & 1420 & 925 & 1000 & 950 & 850 & 780 & 930 & 940 & 1140 & 820 & 1000 & 1345 & 1385 & 900 & 910 & 750 & 700 & 710 & 540 & 930 & 850 & 900 & 620 & 760
\end{tabular}

$\begin{array}{llllllllllllllllllllllllllll}50 & 25 & 25 & 25 & 25 & 50 & 50 & 25 & 25 & 25 & 50 & 50 & 25 & 25 & 25 & 25 & 50 & 50 & 50 & 25 & 25 & 25 & 25 & 25 & 25 & 50 & 25 & 50\end{array}$

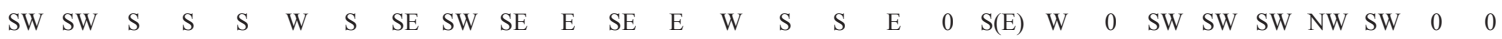

$\begin{array}{lllllllllllllllllllllllllllll}20 & 15 & 15 & 20 & 25 & 15 & 10 & 30 & 20 & 15 & 5 & 30 & 30 & 20 & 25 & 15 & 20 & 0 & 40 & 15 & 0 & 30 & 5 & 20 & 30 & 20 & 0 & 0\end{array}$

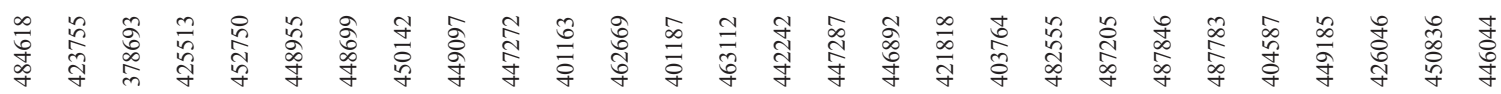

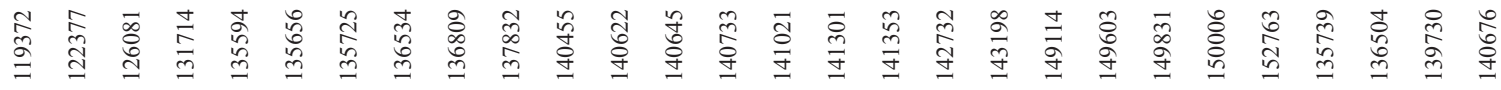

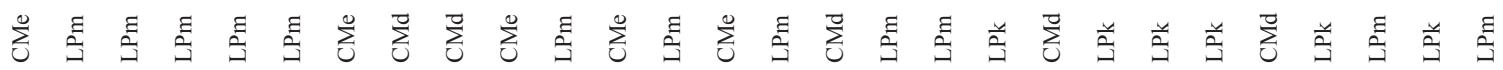

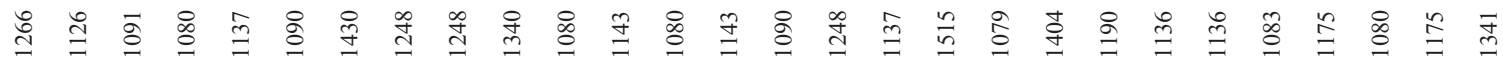

$\begin{array}{llllllllllllllllllllllllllll}100 & 100 & 100 & 100 & 100 & 100 & 100 & 100 & 100 & 100 & 100 & 100 & 100 & 100 & 100 & 100 & 100 & 100 & 100 & 100 & 100 & 100 & 100 & 100 & 100 & 100 & 100 & 100\end{array}$

\begin{tabular}{lllllllllllllllllllllllllllll}
57 & 56 & 56 & 54 & 45 & 62 & 65 & 55 & 49 & 49 & 37 & 37 & 62 & 58 & 61 & 47 & 55 & 53 & 44 & 57 & 35 & 67 & 49 & 64 & 55 & 49 & 43 & 58 & 2 \\
\hline
\end{tabular}

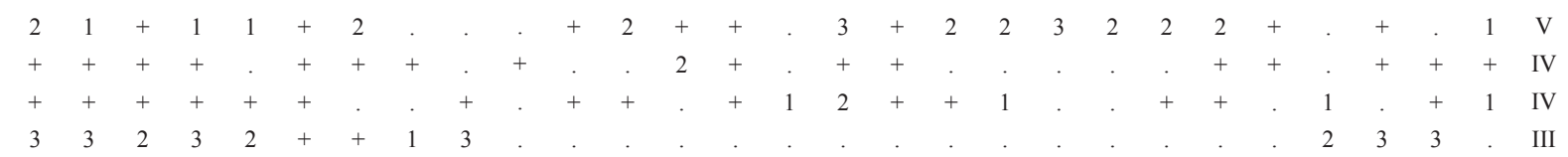

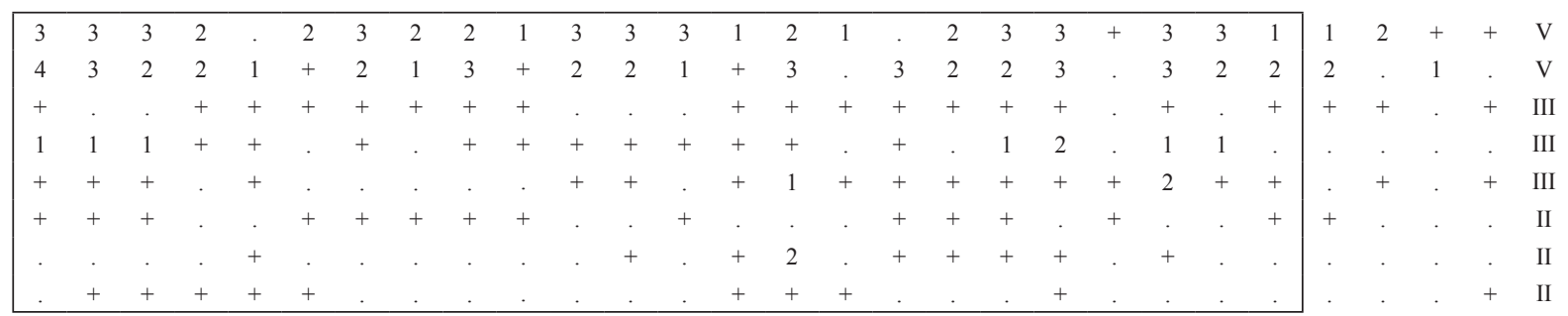

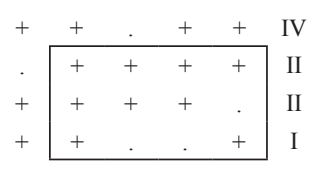


Number of relevé (Številka popisa)

Ranunculus acris ssp. acris

Pastinaca sativa

RANUACRI

\begin{tabular}{llllllllllllllll}
1 & 2 & 3 & 4 & 5 & 6 & 7 & 8 & 9 & 10 & 11 & 12 & 13 & 14 & 15 & 16 \\
\hline
\end{tabular}

PASTSATI

Arrhenatherion Koch 1926

Galium album

GALIALBU

Pimpinella major

Equisetum arvense

PIMPMAJO

Daucus carota

EQUIARVE

Geranium pratense

DAUCCARO

GERAPRAT

Arrhenatheretalia R. Tx. 1931

Stellaria graminea

Helictotrichon pubescens

Colchicum autumnale

Holcus lanatus

Crepis biennis

Medicago lupulina

Heracleum sphondylium

Cynosurus cristatus

Alopecurus pratensis

Aquilegia vulgaris

Rumex obtusifolius

Bromus hordeaceus

STELGRAM

HELIPUBE

COLCAUTU

HOLCLANA

CREPBIEN

MEDILUPU

HERASPON

CYNOCRIS

ALOPPRAT

AQUIVULG

RUMEOBTU

BROMHORD

Cynosurion R. Tx. 1947

Bellis perennis

BELLPERE

Prunella vulgaris

Veronica chamaedrys

Phleum pratense

Trifolium repens

Capsella bursa-pastoris

Erigeron annuus

PRUNVULG

VEROCHAM

PHLEPRAT

TRIFREPE

CAPSBUPA

ERIGANNU

Lolium perenne

LOLIPERE

Molinion Koch 1926

Parnassia palustris

PARNPALU

Senecio integrifolius

SENEINTE

Gentiana asclepiadea

GENTASCL

PRIMVERI

Primula veris

Serratula tinctoria

SERRTINC

Molinietalia Koch 1926

Betonica officinalis

BETOOFFI

Molinia caerulea

MOLICAER

Succisa pratensis

SUCCPRAT

Molinio-Arrhenatheretea R. Tx. 1937 em. R. Tx. 1970

Trisetum flavescens

Dactylis glomerata

Leucanthemum ircutianum

Achillea millefolium

Centaurea jacea ssp. jacea

Lotus corniculatus

Knautia drymeia

Vicia cracca

Tragopogon pratensis ssp. orientalis

Lathyrus pratensis

TRISFLAV DACTGLOM LEUCIRCU ACHIMILL

CENTJACE

LOTUCORNI

KNAUDRYM

VICICRAC

TRAGPRAT

LATHPRAT

$\begin{array}{llllllllllllllll}+ & + & 4 & 3 & 4 & 4 & 3 & 3 & 4 & . & 3 & 4 & 3 & 2 & 4 & 4 \\ + & + & 1 & 2 & 2 & 2 & 1 & 1 & 2 & 1 & 1 & 2 & 1 & 1 & 2 & 1 \\ 3 & + & 2 & 1 & 2 & 2 & 2 & 2 & . & 2 & + & 1 & 2 & 2 & + & 2 \\ 1 & + & + & 1 & + & 1 & 1 & 1 & + & \cdot & + & + & . & 1 & 1 & + \\ + & + & \cdot & \cdot & + & + & + & . & + & + & + & + & + & . & + & + \\ \cdot & + & + & + & + & + & + & \cdot & + & 1 & . & + & + & + & + & + \\ + & \cdot & + & + & + & + & + & + & + & . & + & + & + & . & + & + \\ + & \cdot & \cdot & + & \cdot & + & + & + & + & + & + & + & + & + & + & + \\ + & + & \cdot & \cdot & + & + & \cdot & + & + & + & + & + & + & . & + & + \\ + & \cdot & + & \cdot & + & . & . & + & . & + & . & + & + & + & + & +\end{array}$


Number of relevé (Številka popisa)

Trifolium pratense

Plantago lanceolata

Leontodon hispidus

Festuca rubra

Euphrasia rostkoviana

Taraxacum officinale

Festuca pratensis

Deschampsia cespitosa

Rumex acetosa

Poa pratensis

Poa trivialis

Holcus mollis

Ajuga reptans

\begin{tabular}{lllllllllllllllll} 
& 1 & 2 & 3 & 4 & 5 & 6 & 7 & 8 & 9 & 10 & 11 & 12 & 13 & 14 & 15 & 16 \\
\hline TRIFPRAT &. & 2 & 2 &. & + & + & 1 &. & 1 & 2 & + &. &. & 1 & 1 & + \\
PLANLANC &. & 1 & 1 &. & 1 & 1 &. &. & 1 &. &. & + &. & + & 2 & + \\
LEONHISP & + & + & + & 1 & 1 & 1 & 1 & 1 & 1 &. & + & 1 &. & 1 &. & + \\
FESTRUBR & 1 & + &. &. &. &. &. &. &. &. &. &. &. &. &. &. \\
EUPHROST &. &. &. &. &. &. &. & + &. & + &. & + &. & + & + &. \\
TARAOFFI &. &. &. &. &. & + &. &. & + & + &. & + &. &. &. &. \\
FESTPRAT & 2 &. &. &. & 1 & 1 &. &. & 1 & 2 &. & 1 &. &. & 1 & + \\
DESCCESP &. &. &. &. &. &. &. &. &. & + & + &. &. &. & + & + \\
RUMEACET &. &. &. &. &. &. &. &. &. &. &. &. &. &. &. &. \\
POAPRAT &. &. &. & + &. &. &. &. &. &. &. &. &. &. &. & + \\
POATRIV &. &. &. &. & 1 & + &. &. & + &. &. & 1 &. &. &. &. \\
HOLCMOLL &. &. &. &. &. &. &. &. &. &. &. &. &. &. &. &. \\
AJUGREPT &. &. &. &. &. &. &. &. &. &. &. &. &. &. &. &.
\end{tabular}

Mesobromion Br.-Bl. et Moor 38 em. Oberd. 49 Anthyllis vulneraria

Prunella grandiflora

Thymus pulegioides

Plantago media

Polygala vulgaris

Carex flacca

Knautia arvensis

Carlina acaulis

Silene vulgaris

Campanula glomerata

Inula salicina

Acinos alpinus

Valeriana officinalis

Gentianella germanica

Melica nutans

Carlina vulgaris

Origanum vulgare

Veronica teucrium

Galium lucidum

Chamaespartium sagittale

Gentiana cruciata

Trifolium montanum

ANTHVULN

PRUNGRAN

THYMPULE

PLANMEDI

POLYVULG

CAREFLAC

KNAUARVE

CARLACAU

SILEVULG

CAMPGLOM

INULSALI

ACINALPI

VALEOFFI

GENTGERM

MELINUTA

CARLVULG

ORIGVULG

VEROTEUR

GALILUCI

CHAMSAGI

GENTCRUC

TRIFMONT

Festuco-Brometea Br.-Bl. et R. Tx. ex Klika et Hadač 1944

Rhinanthus freynii

Salvia pratensis

Euphorbia cyparissias

Helianthemum ovatum

Centaurea scabiosa

Cuscuta epithymum

Anthericum ramosum

Filipendula vulgaris

Asperula cynanchica

Helianthemum grandiflorum

Sanguisorba minor

Scabiosa columbaria

Carduus defloratus

Cirsium pannonicum

Hieracium praeltum

Linum catharticum

Melica ciliata

Ononis spinosa
RHINALEC

SALVPRAT

EUPHCYPA

HELIOVAT

CENTSCAB

CUSCEPIT

ANTHRAMO

FILIVULG

ASPRCYNA

HELIGRAN

SANGMINO

SCABCOLU

CARDDEFLO

CIRSPANN

HIERPRAE

LINUCATH

MELICILI

ONONSPIN 
Tina Petras Sackl et all.: Plant Communities with yellow oat grass (Trisetum Flavescens (L.) Pв.) ...

\begin{tabular}{|c|c|c|c|c|c|c|c|c|c|c|c|c|c|c|c|c|c|c|c|c|c|c|c|c|c|c|c|c|}
\hline & 18 & 19 & 20 & 21 & 22 & 23 & 24 & 25 & 26 & 27 & 28 & 29 & 30 & 31 & 32 & 33 & 34 & 35 & 36 & 37 & 38 & 39 & 40 & 41 & 42 & 43 & 44 & Pres \\
\hline . & . & 1 & . & . & 3 & . & 3 & . & 2 & . & . & + & . & 2 & 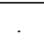 & . & + & . & 1 & 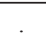 & 1 & 2 & 2 & + & . & . & + & III \\
\hline . & . & 2 & + & . & . & . & + & & + & + & + & + & & 1 & & & . & & + & & 1 & 3 & + & . & 1 & . & + & II \\
\hline+ & 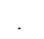 & . & 1 & . & + & + & . & 1 & . & . & . & + & 1 & 1 & . & . & + & + & . & . & + & . & + & 1 & 1 & 1 & 1 & II \\
\hline . & . & + & & . & 1 & + & + & & + & . & + & . & & . & 1 & 2 & + & 2 & 3 & + & + & . & 2 & . & . & . & . & II \\
\hline . & . & + & . & . & + & + & . & . & . & . & . & + & . & . & . & + & + & . & . & . & + & . & . & . & + & . & . & II \\
\hline+ & . & . & . & . & + & . & + & . & . & . & . & . & . & + & . & . & . & . & . & . & . & . & . & . & . & . & . & I \\
\hline . & . & 2 & & . & + & 1 & 1 & & 1 & . & . & . & 2 & 2 & . & . & . & & . & . & . & . & 1 & . & 1 & . & 1 & I \\
\hline . & . & . & . & 1 & . & . & . & . & . & . & . & + & . & . & . & + & . & . & . & . & . & . & . & . & . & . & . & I \\
\hline . & . & . & & . & . & . & + & & + & $\cdot$ & . & . & + & . & . & . & + & & . & . & + & . & . & . & . & . & . & I \\
\hline . & . & . & . & . & . & . & 1 & . & . & . & . & + & 1 & . & 1 & . & . & . & . & . & . & . & 1 & . & . & . & 1 & \\
\hline . & . & . & . & . & . & . & . & . & 2 & . & . & . & . & . & . & . & . & . & & . & . & . & 2 & . & 1 & . & . & + \\
\hline . & . & . & . & . & . & . & 2 & . & + & . & . & . & . & . & . & . & . & . & . & . & . & . & 1 & . & . & . & . & + \\
\hline & . & . & . & . & + & & . & . & . & & . & . & . & . & & & + & & & & & . & . & & & & . & \\
\hline
\end{tabular}

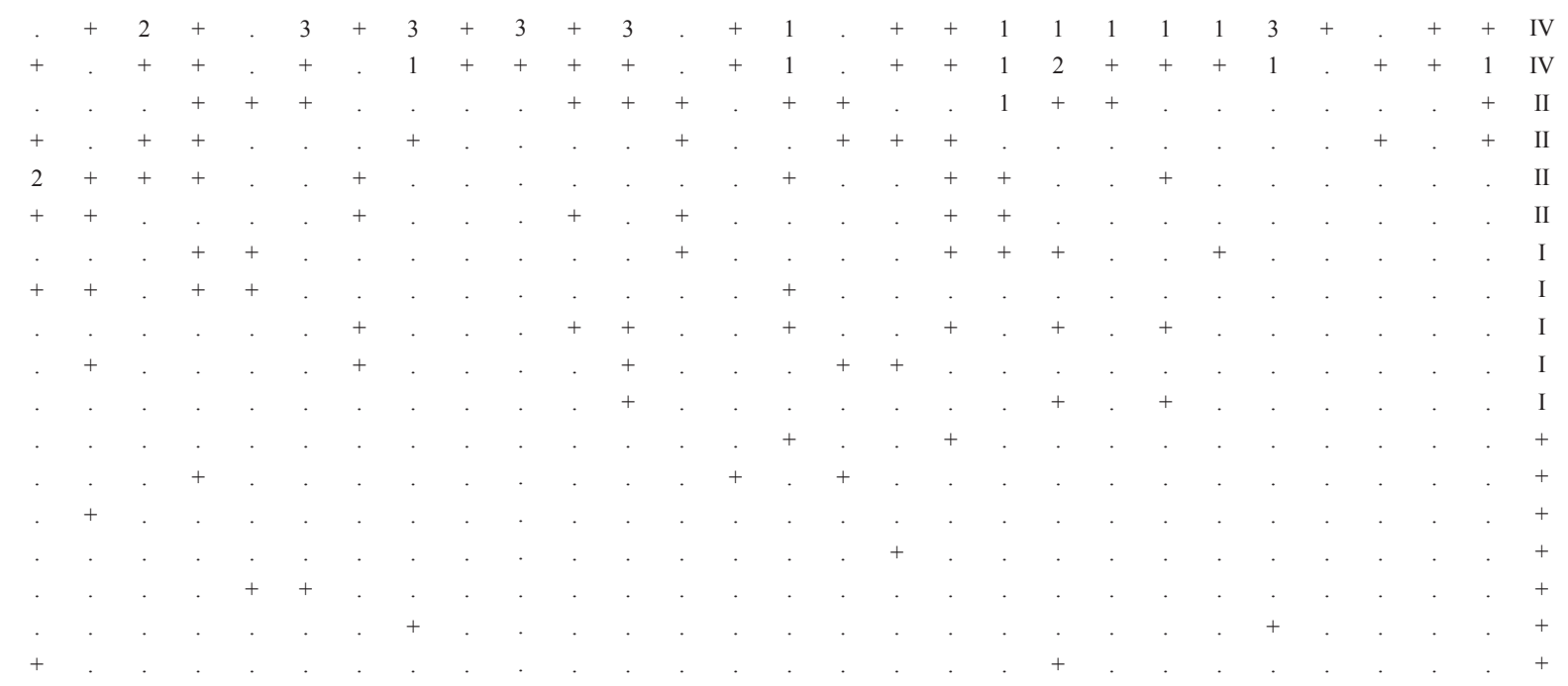


Number of relevé (Številka popisa)

$\begin{array}{llllllllllllllll}1 & 2 & 3 & 4 & 5 & 6 & 7 & 8 & 9 & 10 & 11 & 12 & 13 & 14 & 15 & 16\end{array}$

Nardion Br.-B1. 1926

Polygonum viviparum

POLYVIVI

Leontodon helveticus

LEONHELV

Campanula barbata

CAMPBARB

Ranunculus carinthiacus

RANUCARI

Calluno-Ulicetea Br.-Bl. et R. Tx. ex Klika et Hadač 1944

Anthoxanthum odoratum

ANTHODO

Rumex acetosella

Hypericum maculatum

RUMEACET

Arnica montana

HYPEMACU

Carex pallescens

ARNIMONT

Dactylorhiza maculata

CAREPALL

Hypericum perforatum

DACTMACU

Carex pilulifera

HYPEPERF

Nardus stricta

CAREPILU

Antennaria dioica

NARDSTRI

Calluna vulgaris

ANTEDIOI

CALLVULG

Seslerietalia coeruleae Br.-B1. in Br.-Bl. et Jenny 1926

Betonica alopecuros

BETOALOP

Phyteuma orbiculare

PHYTORBI

Trifolio-Geranietea sanguinei T. Müller 1961

Trifolium medium

Orobanche $s p$.

TRIFMEDI

OROBSP

Lilium bulbiferum

LILIBULB

Lilium carniolicum

LILICARN

Vaccionio-Piceetea Br.-Bl. 1939 em. Pass. 63

Deschampsia flexuosa

Luzula luzuloides

DESCFLEX

LUZULUZU

MELAPRAT

Melampyrum pratense

MAIABIFO

Maianthemum bifolium

Calamagrostis arundinacea

CALAARUN

Luzula luzulina

LUZULUZU

Origanetalia Th. Müller 61

Clinopodium vulgare

CLINVULG

Laserpitium latifolium

Carex spicata

Polygonatum odoratum

Trifolium rubens

LASELATI

CARESPIC

POLYODOR

TRIFRUBE

\section{Other species}

Cruciata glabra

Biscutella laevigata

Alchemilla xanthochlora

Phyteuma zahlbruckneri

Primula vulgaris

Achillea sp.

Tofieldia calyculata

Sedum sp.

Silene nutans

Astrantia carniolica

Scabiosa triandra

Vincetoxicum hirundinaria

CRUCGLAB

BISCLAEV

ACHEXANT

PHYTZAHL

PRIMVULG

ACHISP

TOFICALY

SEDUM

SILENUTA

ASTRCARN

SCABTRIA

VINCHIRU 

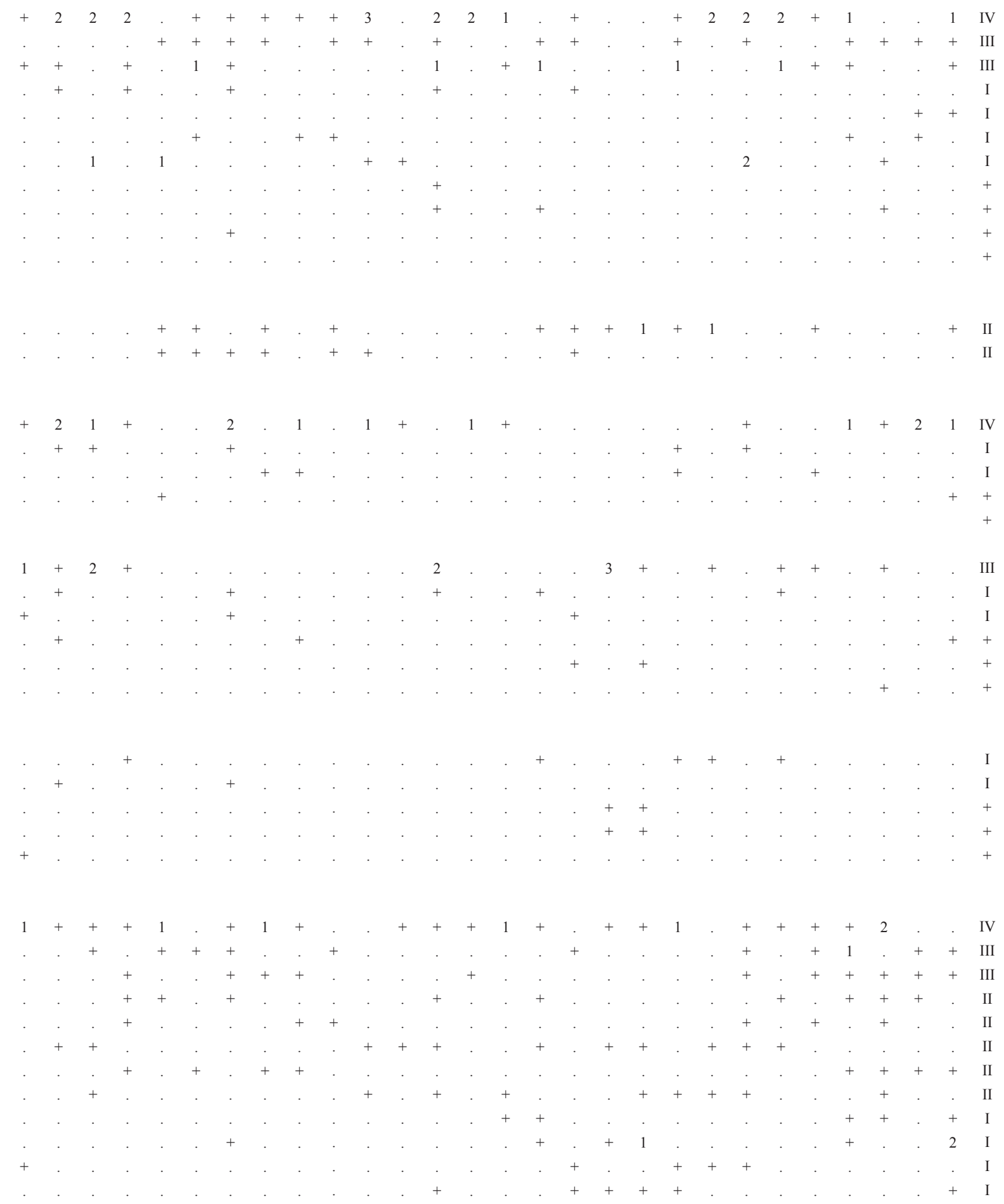
Number of relevé (Številka popisa)

Petrorhagia saxifraga

PETRSAXI

Thalictrum aquilegiifolium

THALAQUI

Trifolium campestre

TRIFCAMP

Campanula trachelium

CAMPTRACH

Helleborus niger

Centaurea phrygia ssp. pseudophrygia

HELLNIG

Aquilegia sp.

CENTPHRY

Vicia sepium

AQUIESP

Viola tricolor

VICISEPI

VIOLTRIC

Alchemilla glabra

ALCHGLAB

Phyteuma ovatum

PHYTOVAT

Chamaecytisus supinus

CHAMSUPI

Rhinanthus glacialis

RHINGLAC

Cirsium eriophorum

CIRSERIO

Table 2: Analytical table of the association Pastinaco-Arrhenatheretum Passarge 1964.

Tabela 2: Analitična tabela asociacije Pastinaco-Arrhenatheretum Passarge 1964.

Number of relevé (Številka popisa)

Altitude (m) (Nadmorska višina)

\begin{tabular}{|c|c|c|c|c|c|c|c|c|c|c|c|c|c|c|c|c|c|}
\hline & 2 & 3 & 4 & 5 & 6 & 7 & 8 & 9 & 10 & 11 & 12 & 13 & 14 & 15 & 16 & 17 & 18 \\
\hline 66 & 470 & 800 & 740 & 690 & 650 & 1000 & 900 & 690 & 1100 & 1100 & 1000 & 500 & 520 & 500 & 507 & 830 & 620 \\
\hline 25 & 25 & 25 & 25 & 25 & 25 & 25 & 25 & 25 & 50 & 25 & 25 & 50 & 25 & 50 & 25 & 50 & 50 \\
\hline W & S & SW & S & S & S & S & $S$ & S & E & S & S & 0 & 0 & 0 & 0 & 0 & SW \\
\hline 30 & 20 & 10 & 10 & 5 & 40 & 5 & 15 & 10 & 20 & 35 & 30 & 0 & 0 & 0 & 0 & 0 & 8 \\
\hline 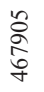 & \begin{tabular}{l}
0 \\
$\stackrel{2}{0}$ \\
\multirow{\sim}{f}{} \\
\end{tabular} & 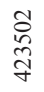 & $\frac{\stackrel{a}{n}}{\tilde{\sigma}}$ & $\begin{array}{l}\infty \\
\text { N } \\
\tilde{y} \\
\text { J }\end{array}$ & $\begin{array}{l}0 \\
\stackrel{0}{0} \\
\dot{q}\end{array}$ & $\begin{array}{l}\hat{\sigma} \\
\frac{\sigma}{\sigma}\end{array}$ & $\begin{array}{l}\infty \\
\infty \\
\infty \\
\infty \\
\infty \\
+\end{array}$ & 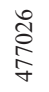 & 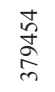 & 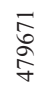 & 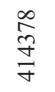 & 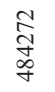 & \begin{tabular}{l}
0 \\
$\stackrel{2}{ }$ \\
$\infty$ \\
\multirow{J}{*}{}
\end{tabular} & 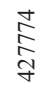 & 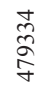 & 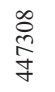 & 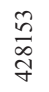 \\
\hline & $\begin{array}{l}\infty \\
\stackrel{\Xi}{\Xi} \\
\stackrel{0}{0}\end{array}$ & $\begin{array}{l}\text { ஜి } \\
\stackrel{8}{0}\end{array}$ & $\begin{array}{l}\stackrel{\cong}{n} \\
\stackrel{\Xi}{\Xi}\end{array}$ & $\stackrel{n}{=}$ & 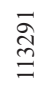 & $\begin{array}{l}\stackrel{2}{\sigma} \\
\stackrel{+}{=}\end{array}$ & 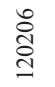 & $\begin{array}{l}\stackrel{a}{\Xi} \\
\underset{J}{ \pm}\end{array}$ & $\begin{array}{l}\widehat{E} \\
\text { స్ } \\
\text { In }\end{array}$ & 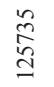 & 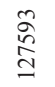 & 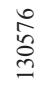 & $\frac{\infty}{\stackrel{\Omega}{~}}$ & $\frac{\check{\sigma}}{\check{g}}$ & 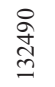 & $\begin{array}{l}\stackrel{2}{0} \\
\text { N } \\
\end{array}$ & 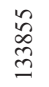 \\
\hline & $\sum_{i}^{\vec{j}}$ & $\sum_{i}^{0}$ & $\sum_{i}^{\vec{j}}$ & $\sum_{i}^{\vec{n}}$ & $\sum_{i}^{\vec{j}}$ & $\sum_{i}^{\vec{n}}$ & $\sum_{j}^{0}$ & $\sum_{i}^{\vec{j}}$ & 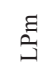 & $\sum_{U}^{\vec{U}}$ & છ & $\sum_{U}^{\vec{U}}$ & $\sum_{i}^{\infty}$ & $\frac{\breve{a}}{\vec{G}}$ & $\sum_{0}^{\vec{U}}$ & $\sum_{0}^{\infty}$ & $\sum_{i}^{0}$ \\
\hline & $\stackrel{\Re}{\Xi}$ & $\stackrel{\ominus}{\Xi}$ & $\stackrel{\varrho}{\Xi}$ & $\stackrel{n}{\Xi}$ & $\stackrel{\varrho}{\Xi}$ & $\stackrel{n}{\Xi}$ & $\begin{array}{l}\stackrel{0}{\circ} \\
\stackrel{1}{0}\end{array}$ & $\stackrel{\varpi}{ٍ}$ & बे & ્ֻ & $\stackrel{n}{n}$ & ฮิ & $\hat{g}$ & ڤે & ปิ & f & $\stackrel{\circ}{\sim}$ \\
\hline & 100 & 100 & 100 & 100 & 100 & 100 & 100 & 100 & 100 & 100 & 100 & 100 & 100 & 100 & 95 & 100 & 100 \\
\hline & 47 & 39 & 31 & 32 & 34 & 34 & 33 & 27 & 36 & 29 & 30 & 35 & 26 & 34 & 33 & 31 & 41 \\
\hline
\end{tabular}

Relevé area (m) (Velikost popisne ploskve)

Exposition (Lega)

Inclination $\left({ }^{\circ}\right)$ (Naklon pobočja)

$\mathrm{X}$-coordinate

(X-koordinata)

Y-coordinate

(Y-koordinata)

Pedologic cartographic unit (Pedološka

kartografska enota - FAO)

Pedologic cartographic unit (Pedološka

kartografska enota - PKE)

Cover (\%) (Pokrovnost)

Number of species (Število vrst)

$\begin{array}{llllllllllllllll}1 & 2 & 3 & 4 & 5 & 6 & 7 & 8 & 9 & 10 & 11 & 12 & 13 & 14 & 15 & 16\end{array}$

Characteristic and differentiating species of the association

Campanula patula

Pastinaca sativa
CAMPPATU

PASTSATI

Characteristic and differentiating species of the subassociation typicum Passarge 1964

Arrhenatherum elatius

Holcus lanatus

Stellaria graminea

Ranunculus acris ssp. acris

Heracleum sphondylium

\begin{tabular}{l|llllllllllllllllllll} 
ARRHELAT & 4 & 3 & 4 & 4 & 4 & 2 & 1 & 4 & 4 & 3 & 3 & & 4 & 4 & 4 & 3 & 4 & 4
\end{tabular}

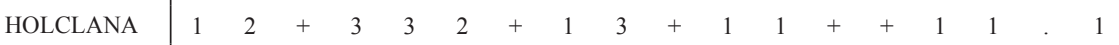

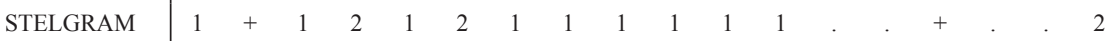

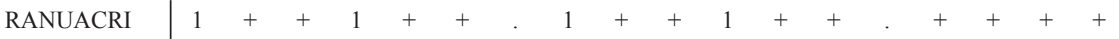

HERASPHO

Characteristic and differentiating species of the subassociation medicagetosum lupulinae Passarge 1964

Anthoxanthum odoratum

Briza media

Salvia pratensis

Knautia arvensis

Pimpinella saxifraga

Medicago lupulina

Galium verum ssp. verum

Bromopsis erecta

\begin{tabular}{|c|c|c|c|c|c|c|c|c|c|c|c|c|c|c|c|c|c|}
\hline ANTHODO & 2 & 2 & + & 2 & 1 & 3 & 2 & 3 & 1 & 1 & 1 & 1 & . & + & . & & . \\
\hline BRIZMEDI & 3 & 3 & 3 & 3 & 3 & 2 & 3 & 2 & 3 & 1 & 1 & 2 & . & . & + & & + \\
\hline SALVPRAT & . & + & + & . & . & . & + & + & . & . & . & . & + & + & + & & + \\
\hline KNAUARVE & 1 & + & . & + & + & + & . & . & . & + & . & . & + & + & . & & . \\
\hline PIMPSAXI & + & + & + & . & + & + & + & . & + & . & . & . & + & + & . & . & . \\
\hline MEDILUPU & . & . & . & . & . & . & . & 1 & . & . & . & . & + & + & . & & + \\
\hline GALIVERU & . & + & + & + & . & . & . & . & + & + & . & . & . & . & + & & . \\
\hline BROMEREC & + & & . & . & . & + & . & . & . & . & . & . & . & . & + & & . \\
\hline
\end{tabular}


\begin{tabular}{ccccccccccccccccccccccccccccc}
19 & 20 & 21 & 22 & 23 & 24 & 25 & 26 & 27 & 28 & 29 & 30 & 31 & 32 & 33 & 34 & 35 & 36 & 37 & 38 & 39 & 40 & 41 & 42 & 43 & 44 & 45 & 46 & 47 \\
\hline 615 & 770 & 1000 & 600 & 680 & 440 & 700 & 800 & 560 & 535 & 680 & 530 & 760 & 550 & 693 & 540 & 530 & 480 & 530 & 400 & 500 & 660 & 600 & 500 & 605 & 640 & 625 & 745 & 750
\end{tabular} $\begin{array}{lllllllllllllllllllllllllllll}25 & 25 & 25 & 25 & 25 & 25 & 25 & 50 & 25 & 25 & 25 & 25 & 25 & 25 & 25 & 25 & 50 & 50 & 25 & 50 & 50 & 25 & 50 & 25 & 25 & 25 & 25 & 25 & 50\end{array}$ $\begin{array}{llllllllllllllllllllllllllllllllllllll}\text { SW } & \text { S } & \text { SW } & 0 & \text { SE } & \text { NE } & \text { S } & \text { S } & \text { W } & 0 & \text { S } & (\mathrm{S}) W & \text { W } & 0 & \text { SE } & \text { S } & 0 & \text { S } & \text { S } & 0 & \text { S } & 0 & \text { E } & \text { SE } & \text { SE } & \text { SW } & \text { W } & \text { S } & \text { W }\end{array}$ $\begin{array}{lllllllllllllllllllllllllllllllllllllllll}3 & 10 & 5 & 0 & 15 & 20 & 25 & 35 & 20 & 0 & 20 & 2 & 30 & 0 & 10 & 15 & 0 & 30 & 10 & 0 & 5 & 0 & 15 & 20 & 8 & 10 & 15 & 5 & 8\end{array}$

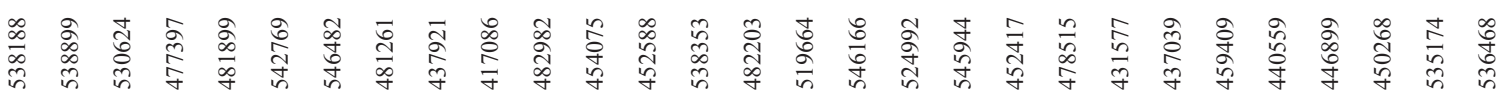

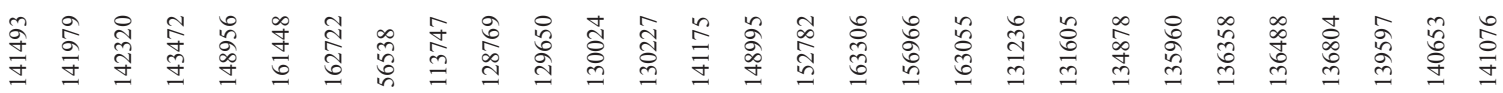

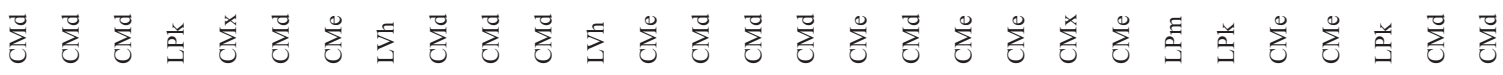

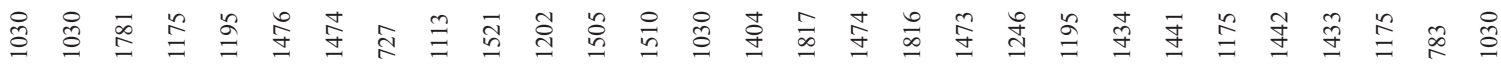
$\begin{array}{llllllllllllllllllllllllllllll}100 & 100 & 100 & 100 & 100 & 100 & 100 & 100 & 100 & 100 & 98 & 100 & 100 & 100 & 100 & 100 & 100 & 100 & 100 & 95 & 98 & 100 & 100 & 100 & 100 & 100 & 100 & 100 & 100\end{array}$ $\begin{array}{llllllllllllllllllllllllllllll}34 & 25 & 33 & 29 & 34 & 34 & 28 & 48 & 36 & 35 & 40 & 42 & 50 & 54 & 34 & 42 & 30 & 39 & 31 & 26 & 33 & 37 & 37 & 32 & 40 & 38 & 32 & 40 & 36 & \mathbf{2}^{2}\end{array}$

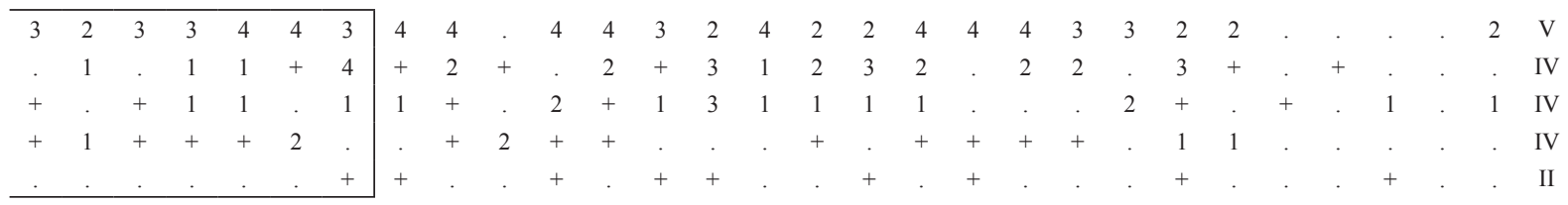

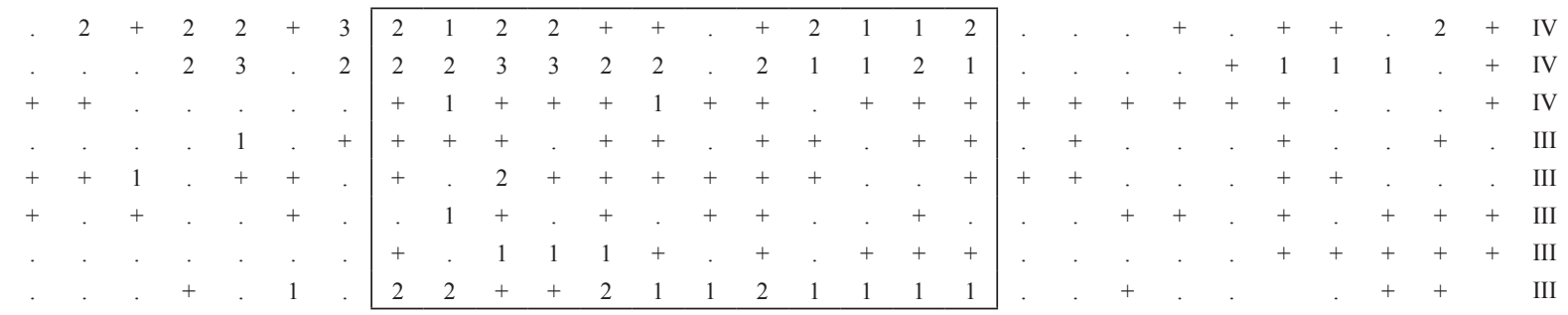


Number of relevé (Številka popisa)

\begin{tabular}{llllllllllllllllll}
1 & 2 & 3 & 4 & 5 & 6 & 7 & 8 & 9 & 10 & 11 & 12 & 13 & 14 & 15 & 16 & 17 & 18 \\
\hline
\end{tabular}

Characteristic and differentiating species of the subassociation lolietosum subass. nova

Prunella vulgaris

PRUNVULG

Capsella bursa-pastoris

CAPSBUPA

Erigeron annuus

ERIGANNU

Lolium perenne

LOLIPERE

Phleum pratense

PHLEPRAT

Arrhenatherion Koch 1926

Galium album

GALIALBU

Daucus carota

Pimpinella major

DAUCCARO

Equisetum arvense

PIMPMAJO

Medicago sativa

EQUIARVE

Potentilla reptans

MEDISATI

POTEREPT

Arrhenatheretalia R. Tx. 1931

Crepis biennis

CREPBIEN

Cynosurus cristatus

Helictotrichon pubescens

CYNOCRIS

HELIPUBE

Alopecurus pratensis

ALOPPRAT

Rumex obtusifolius

RUMEOBTU

Colchicum autumnale

COLCAUTU

Lychnis flos-cuculi

LYCHFLCU

Bromus hordeaceus

BROMHORD

Astrantio-Trisetetum Knapp et Knapp ex Oberd. 1957

Luzula campestris

Trifolium alpestre

LUZUCAMP

Potentilla erecta

TRIFALPE

POTEEREC

Polygono-Trisetion Br.-Bl. et R. Tx. ex Marschall 1947 nom. inv.

Geranium sylvaticum

Ranunculus nemorosus

Crocus vernus

Cynosurion R. Tx. 1947

Bellis perennis

Veronica chamaedrys

Plantago major

Ranunculus repens

Molinietalia Koch 1926

Cirsium oleraceum

Betonica officinalis

Molinia caerulea

Symphytum officinale
GERASYLV

RANUNEMO

CROC VERN

Molinio-Arrhenatheretea R. Tx. 1937 em. R. Tx. 1970

Trisetum flavescens

Plantago lanceolata

Dactylis glomerata

Trifolium medium

Lotus corniculatus

Trifolium pratense

Knautia drymeia

Vicia cracca

Leucanthemum ircutianum
BELLPERE VEROCHAM

PLANMAJO

RANUREPE

CIRSOLER BETOOFFI

MOLICAER

SYMPOFFI

$\begin{array}{lccccccccccccccccccc}\text { TRISFLAV } & 3 & 4 & 4 & 4 & 4 & 4 & 4 & 4 & 4 & 3 & 5 & 4 & 4 & 4 & 4 & 5 & 4 & 4 \\ \text { PLANLANC } & + & + & 1 & 1 & 1 & 1 & + & + & 1 & . & + & + & 2 & 1 & + & 1 & 2 & + \\ \text { DACTGLOM } & 2 & 1 & 1 & 1 & . & 2 & 1 & 2 & . & + & 1 & 2 & + & 1 & 2 & + & + & + \\ \text { TRIFMEDI } & 2 & . & 1 & + & + & 1 & 2 & 1 & . & + & 1 & + & 2 & 3 & 1 & . & + & 1 \\ \text { LOTUCORNI } & 1 & + & + & + & + & 1 & 2 & + & . & + & 1 & . & 1 & + & 1 & + & + & 1 \\ \text { TRIFPRAT } & . & . & . & . & 1 & 1 & + & 1 & 2 & 2 & 1 & 2 & 3 & 1 & 2 & + & 3 & 2 \\ \text { KNAUDRYM } & + & + & + & + & + & + & + & + & + & + & + & + & 1 & + & + & + & . & + \\ \text { VICICRAC } & + & + & + & + & . & . & + & + & . & + & + & + & + & + & . & + & + & + \\ \text { LEUCIRCU } & 2 & + & 2 & 1 & 2 & 2 & 2 & 3 & . & 2 & + & 2 & 2 & 1 & + & 2 & 1 & +\end{array}$




\begin{tabular}{|cccccccccc|c|}
\hline+ & + & 1 & + & 1 & + & + & 1 & + & 1 & IV \\
$\cdot$ & + & + & + & + &. & + & + &. & + & II \\
+ & + &. &. &. &. &. &. &. &. & II \\
1 & 1 & 3 & 3 & 3 & 1 & + & + & + & 1 & I \\
+ & + &. &. & + & 3 &. &. &. & + & I
\end{tabular}

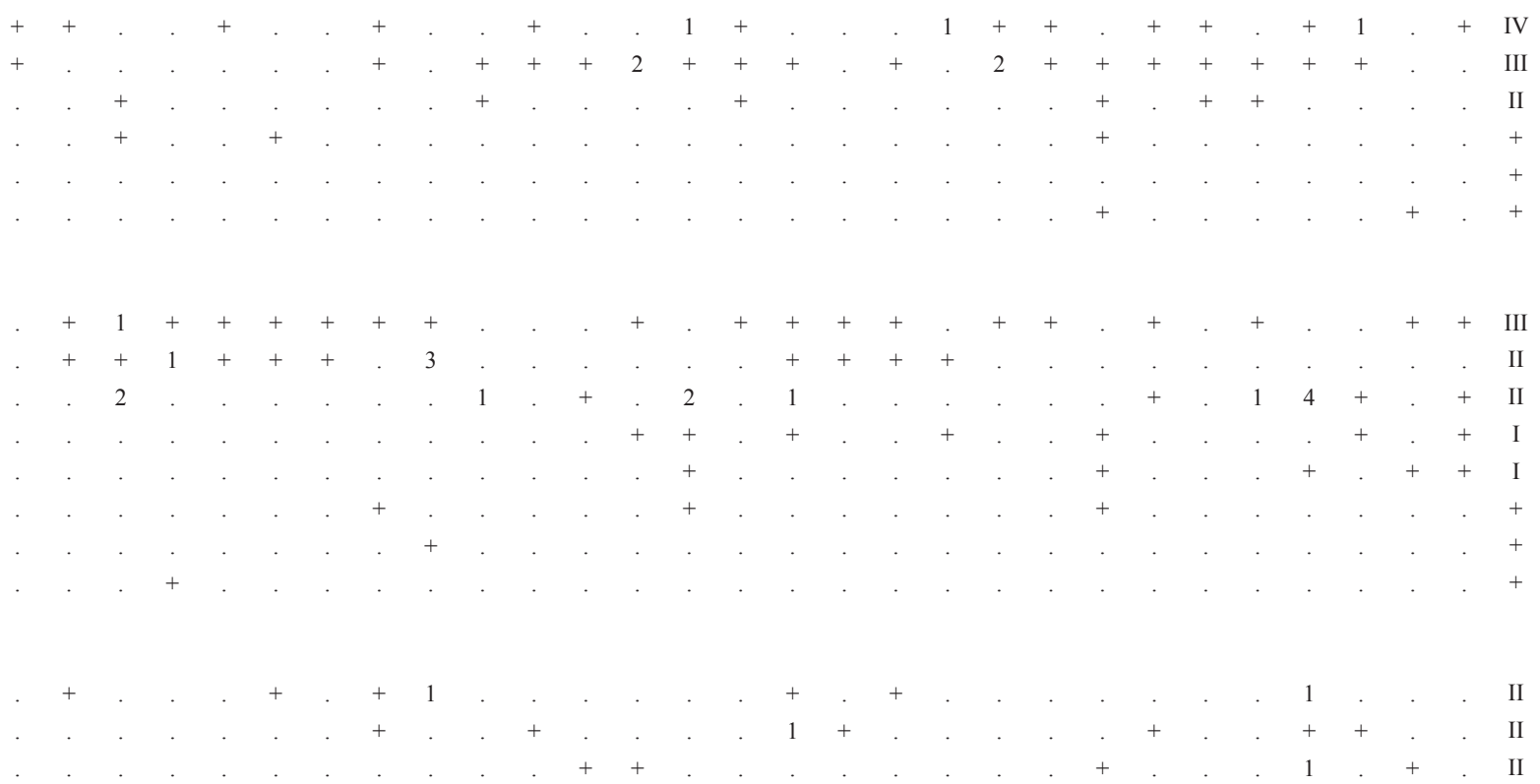

\begin{tabular}{|c|c|c|c|c|c|c|c|c|c|c|c|c|c|c|c|c|c|c|c|c|c|c|c|c|c|c|c|c|c|}
\hline & 3 & 5 & 4 & 4 & 4 & 4 & 4 & 4 & 5 & 3 & + & + & 4 & 4 & 3 & 3 & 4 & 4 & 4 & 4 & 4 & 4 & 4 & + & 4 & 5 & 4 & 1 & $\mathrm{~V}$ \\
\hline & 1 & . & + & + & . & 1 & + & . & 2 & 2 & + & + & 2 & 1 & 1 & 1 & 1 & 1 & 1 & 1 & 3 & 3 & 2 & 1 & 2 & 2 & + & 2 & $\mathrm{~V}$ \\
\hline & 2 & + & 1 & 1 & 2 & 1 & 1 & + & 2 & 2 & 1 & 2 & 1 & 3 & 1 & 1 & 2 & + & + & + & . & . & + & 2 & 1 & & 1 & 1 & $\mathrm{~V}$ \\
\hline & . & 2 & 3 & 2 & . & 1 & 1 & . & 1 & + & + & + & + & . & + & 1 & 1 & 2 & 3 & 1 & + & + & + & 2 & + & 2 & + & + & $\mathrm{V}$ \\
\hline & + & + & . & 1 & + & + & . & + & + & + & + & . & + & . & 1 & + & 1 & + & 1 & + & 1 & 1 & 1 & . & + & + & + & & $\mathrm{V}$ \\
\hline & 3 & 2 & 3 & + & 2 & 3 & 1 & 1 & 2 & + & . & + & + & 2 & 3 & 1 & 3 & . & 1 & 3 & 3 & 2 & 3 & 1 & 3 & 2 & 3 & 3 & $\mathrm{~V}$ \\
\hline & + & + & . & + & + & + & + & + & + & + & + & . & . & + & + & . & + & + & + & + & + & + & + & + & . & + & . & . & $\mathrm{V}$ \\
\hline & + & + & + & + & 1 & + & 1 & 1 & + & . & 1 & + & + & + & + & + & + & + & + & . & + & + & + & + & + & + & + & + & $\mathrm{V}$ \\
\hline & 1 & 1 & 2 & 2 & + & 2 & 2 & 2 & + & 2 & & . & + & + & + & 1 & 1 & 1 & 3 & 2 & + & 1 & 3 & + & + & + & . & & V \\
\hline
\end{tabular}


Number of relevé (Številka popisa)

Centaurea jacea ssp. jacea

Tragopogon pratensis subsp. orientalis

Festuca pratensis

Leontodon hispidus

Achillea millefolium

Lathyrus pratensis

Poa pratensis

Euphrasia rostkoviana

Festuca rubra

Deschampsia cespitosa

Rumex acetosa

Taraxacum officinale

Poa trivialis

Holcus mollis

Ajuga reptans

CENTJACE
TRAGPRAT
FESTPRAT
LEONHISP
ACHIMILL
LATHPRAT
POAPRAT
EUPHROST
FESTRUBR
DESCCESP
RUMEACET
TARAOFFI
POATRIV
HOLCMOLL
AJUGREPT

Mesobromion Br.-B1. et Moor 38 em. Oberd. 49

Silene vulgaris

SILEVULG

Thymus pulegioides

THYMPULE

Salvia verticillata

SALVVERT

Veronica teucrium

VEROTEUR

Brometalia erecti Br.-B1. 1937

Plantago media

PLANMEDI

Anthyllis vulneraria

ANTHVULN

Polygala vulgaris

POLYVULG

Carex flacca

CAREFLAC

Prunella grandiflora

PRUNGRAN

Carlina acaulis

CARLACAU

Chamaespartium sagittale

CHAMSAGI

Gymnadenia conopsea

GYMNCONO

Polygala chamaebuxus

POLYCHAM

Trifolium montanum

TRIFMONT

Campanula glomerata

CAMPGLOM

Festuco-Brometea Br.-B1. et R. Tx. ex Klika et Hadač 1944

Rhinanthus freynii

Sanguisorba minor

Centaurea scabiosa

Allium carinatum

Hieracium praeltum

Ononis spinosa

Filipendula vulgaris

Helianthemum ovatum

Cuscuta epithymum

Euphorbia cyparissias

Anthericum ramosum

Centaurium erythraea

Brachypodium rupestre

Phleum phleoides
RHINALEC

SANGMINO

CENTSCAB

ALLICAARI

HIERPRAE

ONONSPIN

FILIVULG

HELIOVAT

CUSCEPIT

EUPHCYPA

ANTHRAMO

CENTERYT

BRACRUPE

PHLEPHLE

Calluno-Ulicetea Br.-B1. et R. Tx. ex Klika et Hadač 1944

Rumex acetosella

Hypericum perforatum

Hypericum maculatum

Agrostis tenuis

Carex pallescens
RUMEACET

HYPEPERF

HYPEMACU

AGROTENN

CAREPALL

$\begin{array}{lllllll}1 & 2 & 3 & 4 & 5 & 6 & 7 \\ + & + & + & 1 & + & + & +\end{array}$

(1)

10

$\begin{array}{cccccccc}1 & 12 & 13 & 14 & 15 & 16 & 17 & 18 \\ + & + & + & + & 1 & 1 & 2 & 1\end{array}$

$+++1$

$1 .+1$

$\begin{array}{llll}2 & 1 & 1 & 1\end{array}$

$+$

$\begin{array}{lllllll}1 & 1 & 1 & 1 & 1 & 1 & + \\ + & + & + & . & + & + & +\end{array}$

$+++.+++$

$\begin{array}{rr} & + \\ 3 & 1 \\ 2 & \cdot \\ 1 & + \\ + & + \\ + \\ +\end{array}$

$\begin{array}{ll}+ & . \\ 1 & 1 \\ \cdot & . \\ + & 2 \\ + & + \\ 1 & \\ + & \end{array}$

$\cdot$
1
$\cdot$
2
+
$\cdot$
+

+
+
1
2
+
+
+
+

$\begin{array}{ll}+ & \cdot \\ + & 2 \\ 1 & 2 \\ + & + \\ + & \cdot \\ 1 & \cdot \\ \cdot & + \\ \cdot & +\end{array}$

$\begin{array}{rrrrrr}+ & + & + & + & + & + \\ \cdot & \cdot & + & + & \cdot & + \\ 1 & 2 & 2 & 2 & 1 & 2\end{array}$

$\begin{array}{lllll}2 & 1 & 2 & 2 & 1\end{array}$

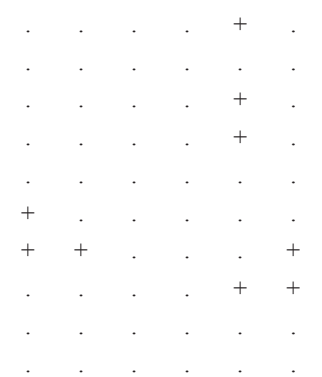

$\cdot$ 
Tina Petras Sackl et all.: Plant Communities with yellow oat grass (TRisetum Flavescens (L.) Pв.) ...
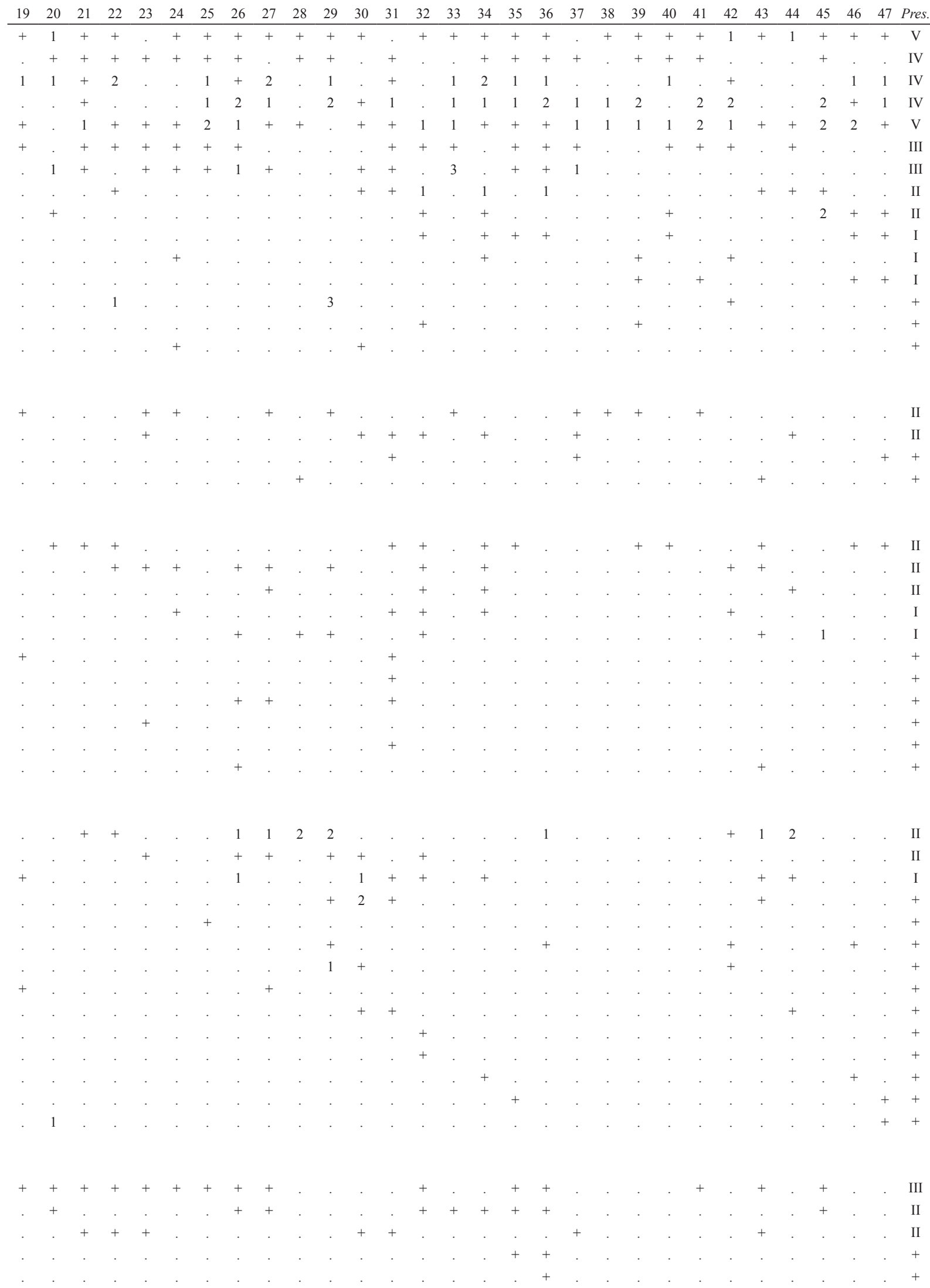
Number of relevé (Številka popisa)

Leontodon helveticus

$\begin{array}{llllllllllllllllll}1 & 2 & 3 & 4 & 5 & 6 & 7 & 8 & 9 & 10 & 11 & 12 & 13 & 14 & 15 & 16 & 17 & 18\end{array}$

Nardus stricta

LEONHELV

NARDSTRI

Origanetalia Th. Müller 61

Silene nutans

SILENUTA

Clinopodium vulgare

Orobanche sp.

Origanum vulgare

CLINVULG

OROBSP

Laserpitium latifolium

ORIGVULG

LASELATI

Secalietea Br-B1. 51

Agropyron repens

Lolium multiflorum

AGROREPE

Poa annua

LOLIMULT

POAANNU

\section{Other species}

Convolvulus arvensis

Cruciata glabra

Trifolium campestre

Alchemilla xanthochlora

Deschampsia flexuosa

Sedum reflexum

Galium lucidum

Achillea sp.

Campanula trachelium

Primula vulgaris

Calamagrostis arundinacea

CONVARVE

CRUCGLAB

TRIFCAMP

ALCHXANT

DESCFLEX

SEDUREFL

GALILUCI

ACHIATRA

CAMPTRAC

PRIMVULG

CALAARUN

PETRSAXI

CIRSARV

CIRSERIO

PHYTZAHL

SCABTRIA

Scabiosa triandra

VACCPYRA 
Tina Petras Sackl et all.: Plant Communities with yellow oat grass (Trisetum Flavescens (L.) Pв.) ...

\begin{tabular}{llllllllllllllllllllllllllllll}
19 & 20 & 21 & 22 & 23 & 24 & 25 & 26 & 27 & 28 & 29 & 30 & 31 & 32 & 33 & 34 & 35 & 36 & 37 & 38 & 39 & 40 & 41 & 42 & 43 & 44 & 45 & 46 & 47 & Pres. \\
\hline
\end{tabular}

\begin{tabular}{|c|c|c|c|c|c|c|c|c|c|c|c|c|c|c|c|c|c|c|c|c|c|c|c|c|c|c|c|c|}
\hline . & . & + & . & + & + & + & + & . & + & + & . & . & . & + & . & . & . & + & + & + & . & + & & . & & $\cdot$ & . & . \\
\hline+ & . & . & . & . & . & . & . & . & . & . & + & + & + & + & + & . & . & . & + & + & . & + & . & . & $\cdot$ & $\cdot$ & . & + \\
\hline . & . & . & . & . & . & . & + & . & . & . & . & . & . & . & . & . & . & . & . & . & . & . & . & $\cdot$ & . & $\cdot$ & . & . \\
\hline . & . & . & . & . & . & . & + & . & . & . & 1 & . & . & . & . & . & . & . & . & . & . & . & . & . & . & $\cdot$ & + & . \\
\hline . & . & . & . & + & . & . & + & . & . & . & . & . & . & . & . & . & . & . & . & . & . & . & . & . & . & . & . & . \\
\hline
\end{tabular}

\begin{tabular}{|c|c|c|c|c|c|c|c|c|c|c|c|c|c|c|c|c|c|c|c|c|c|c|c|c|c|c|c|c|c|}
\hline+ & + & . & . & + & . & + & + & . & . & . & + & + & . & + & . & + & + & . & . & + & . & + & . & . & . & . & + & . & II \\
\hline . & . & . & + & . & . & . & 1 & . & . & + & + & . & + & . & . & . & 1 & . & . & . & . & . & . & + & . & . & . & . & II \\
\hline . & . & . & . & . & . & . & . & . & . & . & . & . & . & . & 2 & . & . & + & . & + & . & . & + & + & . & . & + & . & II \\
\hline . & . & . & . & . & . & . & + & + & . & . & . & . & . & . & . & . & . & . & . & . & . & . & . & . & . & . & . & . & II \\
\hline . & . & . & . & . & . & . & . & . & 2 & . & . & . & + & . & . & 1 & . & . & . & . & 1 & . & . & + & 2 & . & . & . & I \\
\hline . & . & . & . & + & . & . & + & + & . & . & . & + & 1 & . & . & . & . & + & . & . & . & . & . & . & + & . & . & . & I \\
\hline . & . & . & . & . & . & . & . & . & . & . & + & + & . & . & + & . & . & . & . & . & . & . & . & . & . & . & + & + & I \\
\hline+ & . & . & . & . & . & . & . & . & . & 1 & . & . & + & . & . & . & . & . & . & . & + & . & . & . & . & . & . & . & + \\
\hline . & . & . & . & . & . & . & . & . & . & . & . & . & . & + & . & . & + & . & . & . & . & . & . & . & . & . & + & . & + \\
\hline . & . & . & . & . & . & . & + & . & $\cdot$ & + & . & . & . & . & . & . & . & . & . & . & . & . & . & . & . & . & . & . & + \\
\hline . & . & . & . & . & . & . & . & . & . & . & 2 & . & + & . & . & . & . & . & . & . & . & . & . & . & . & . & . & . & + \\
\hline . & . & . & . & . & . & . & + & . & . & . & . & . & + & . & . & . & . & . & . & . & . & . & . & . & . & . & . & . & + \\
\hline . & . & . & . & . & . & . & . & . & . & . & . & . & . & . & . & . & . & . & . & . & . & . & . & + & . & + & . & . & + \\
\hline . & . & . & . & . & . & . & . & . & . & . & . & . & . & . & . & . & . & . & . & . & . & . & . & . & . & . & . & + & + \\
\hline$\cdot$ & . & . & . & . & . & . & . & . & . & . & . & + & . & . & . & . & . & . & . & . & . & . & . & . & . & . & . & . & + \\
\hline . & + & . & . & . & . & . & . & . & . & . & . & . & + & . & . & . & . & . & . & . & . & . & . & . & . & . & . & . & + \\
\hline . & . & . & . & . & . & . & . & . & . & . & . & + & . & . & . & + & . & . & . & . & . & . & . & . & . & . & . & . & + \\
\hline
\end{tabular}


Appendix to the tables 1 and 2: Interpretation of pedological cartographic units.

Dodatek k tabelama 1 in 2: Pojasnilo k pedološkim kartografskim enotam.

\begin{tabular}{|c|c|c|c|c|}
\hline FAO & FAO_TYPE & FAO_TIPI & PKE & PKET \\
\hline $\mathrm{CMe}$ & Eutric Cambisol & CMe_100\% & 216 & EVT RJ / DELUVIJU, KOLUVIALNA_100\% \\
\hline $\mathrm{LVh}$ & Haplic Luvisol & LVh_100\% & 727 & IZPRANA (LUVISOL) / PLIOC SEDIMENTIH, tip_100\% \\
\hline CMd & Dystric Cambisol & CMd_100\% & 783 & DIS RJ / GNAJSU, tip gl_100\% \\
\hline CMd & Dystric Cambisol & CMd_70\%, LPd_30\% & 1030 & DIS RJ/METAMORFNIH kam tip 70\%, RANKER, DIS REGOLITIČNI_30\% \\
\hline LPk & Rendzic Leptosol & LPk_50\%, LPk_50\% & 1079 & $\begin{array}{l}\text { RENDZINA / apn }+ \text { dol PRHNINASTA 50\%, RENDZINA / apn }+ \text { dol } \\
\text { SPRSTENINASTA_50\% }\end{array}$ \\
\hline LPm & Mollic Leptosol & LPm_50\%,LPm_50\% & 1080 & $\begin{array}{l}\text { RENDZINA / MORENI, PRHNINASTA 50\%, RENDZINA / MORENI, } \\
\text { SPRSTENINASTA_50\% }\end{array}$ \\
\hline CMd & Dystric Cambisol & CMd_80\%,CMd_20\% & 1083 & DIS RJ / DELUVIJU, tip 80\%, DIS RJ / DELUVIJU, KOLUVIALNA_20\% \\
\hline LPm & Mollic Leptosol & LPm_70\%, LPq $30 \%$ & 1089 & $\begin{array}{l}\text { RENDZINA / POBOČNEM GRUŠČU, PRHNINASTA 70\%, LITOSOL, } \\
\text { KARB / POBOČNEM GRUŠČU_30\% }\end{array}$ \\
\hline LPm & Mollic Leptosol & LPm_80\%, LPm_20\% & 1090 & $\begin{array}{l}\text { RENDZINA / POBOČNEM GRUŠČU, PRHNINASTA 80\%, RENDZINA / } \\
\text { POBOČNEM GRUŠČU, SPRSTENINASTA_ } 20 \%\end{array}$ \\
\hline LPm & Mollic Leptosol & LPm_80\%,CMe_20\% & 1091 & $\begin{array}{l}\text { RENDZINA / MORENI, SPRSTENINASTA } 80 \% \text {, EVT RJ / MORENI, } \\
\text { tip_20\% }\end{array}$ \\
\hline CMd & Dystric Cambisol & $\begin{array}{l}\text { CMd50\%, CMe_30\%, } \\
\text { CMd_20\% }\end{array}$ & 1093 & $\begin{array}{l}\text { DIS RJ / NENEKARB FLIŠU + DEKALCIFICIRANEM LAPORJU, tip } \\
50 \% \text {, EVT RJ / PALEOCENSKEM + KREDNEM FLIŠU, tip_ } 30 \% \text {, DIS RJ } \\
\text { / NENEKARB FL }\end{array}$ \\
\hline $\mathrm{CMe}$ & Eutric Cambisol & CMe_80\%, LPe_20\% & 1110 & $\begin{array}{l}\text { EVT RJ / MEŠANIH karb + nekarb kam tip } 80 \% \text {, RANKER, EVT } \\
\text { REGOLITIČNI_20\% }\end{array}$ \\
\hline CMd & Dystric Cambisol & CMd_80\%, LPd_20\% & 1113 & $\begin{array}{l}\text { DIS RJ / PERMO-KARBONSKIH skril + pešč tip } 80 \% \text {, RANKER, DIS } \\
\text { EROZIJSKI_20\% }\end{array}$ \\
\hline CMd & Dystric Cambisol & CMd_80\%, LPd_20\% & 1115 & DIS RJ / GRODENSKIH pešč tip 80\%, RANKER, DIS EROZIJSKI_20\% \\
\hline LPm & Mollic Leptosol & LPm_60\%, LPm_40\% & 1126 & $\begin{array}{l}\text { RENDZINA / MORENI, PRHNINASTA } 60 \% \text {, RENDZINA / MORENI, } \\
\text { KOLUVIALNA_40\% }\end{array}$ \\
\hline LPk & Rendzic Leptosol & LPk_70\%, LPk_30\% & 1136 & $\begin{array}{l}\text { RENDZINA / apn }+ \text { dol SPRSTENINASTA } 70 \% \text {, RENDZINA / apn }+ \text { dol } \\
\text { PRHNINASTA_30\% }\end{array}$ \\
\hline LPm & Mollic Leptosol & $\begin{array}{l}\text { LPm60\%, LPm_20\%, } \\
\text { LPm_20\% }\end{array}$ & 1137 & $\begin{array}{l}\text { RENDZINA / POBOČNEM GRUŠČU, SPRSTENINASTA } 60 \% \text {, RENDZI- } \\
\text { NA / POBOČNEM GRUŠČU, PRHNINASTA_20\%, RENDZINA / POBOČ- } \\
\text { NEM GRUŠČU, KOLUVIA }\end{array}$ \\
\hline LPm & Mollic Leptosol & $\begin{array}{l}\mathrm{LPm} 50 \%, \mathrm{LPm}_{-} 30 \% \\
\mathrm{LPm} \_20 \%\end{array}$ & 1138 & $\begin{array}{l}\text { RENDZINA / MORENI, SPRSTENINASTA 50\%, RENDZINA / MORENI, } \\
\text { PRHNINASTA_30\%, RENDZINA / MORENI, SUROVI HUMUS_20\% }\end{array}$ \\
\hline $\mathrm{CMe}$ & Eutric Cambisol & CMe_80\%,CMe_20\% & 1141 & $\begin{array}{l}\text { EVT RJ / LED DOB prod + pešč NASUTINAH REK + REČ VRŠAJU, tip } \\
80 \%, \text { EVT RJ / MORENI, OGLEJENA_ } 20 \%\end{array}$ \\
\hline $\mathrm{CMe}$ & Eutric Cambisol & CMe_80\%,LPe_20\% & 1143 & EVT RJ / DELUVIJU, tip 80\%, RANKER, EVT REGOLITIČNI_20\% \\
\hline $\mathrm{CMe}$ & Eutric Cambisol & CMe_80\%,LPm_20\% & 1151 & $\begin{array}{l}\text { EVT RJ / POBOČNEM GRUŠČU, tip } 80 \% \text {, RENDZINA / POBOČNEM } \\
\text { GRUŠČU, SPRSTENINASTA_20\% }\end{array}$ \\
\hline LPk & Rendzic Leptosol & $\begin{array}{l}\text { LPk70\%, LPk_20\%, } \\
\text { LPk_10\% }\end{array}$ & 1175 & $\begin{array}{l}\text { RENDZINA / apn + dol SPRSTENINASTA 70\%, RENDZINA / apn }+ \text { dol } \\
\text { PRHNINASTA_20\%, RENDZINA / apn }+ \text { dol RJ_10\% }\end{array}$ \\
\hline LPk & Rendzic Leptosol & $\begin{array}{l}\text { LPk } 40 \%, \text { LPk_30\%, } \\
\text { CMx_30\% }\end{array}$ & 1190 & $\begin{array}{l}\text { RENDZINA / apn }+ \text { dol RJ } 40 \% \text {, RENDZINA / apn }+ \text { dol SPRSTENINA- } \\
\text { STA_30\%, RJ POKARB / apn }+ \text { dol tip_30\% }\end{array}$ \\
\hline $\mathrm{CMx}$ & Chromic Cambisol & CMx_70\%, LPk_30\% & 1195 & $\begin{array}{l}\text { RJ POKARB / apn + dol tip 70\%, RENDZINA / apn + dol SPRSTENINA- } \\
\text { STA_30\% }\end{array}$ \\
\hline CMd & Dystric Cambisol & CMd_70\%, LPd_30\% & 1202 & $\begin{array}{l}\text { DIS RJ / PIROKLASTIČNIH kam tip 70\%, RANKER, DIS REGOLITIČNI } \\
\_30 \%\end{array}$ \\
\hline LPd & Dystric Leptosol & $\begin{array}{l}\text { LPd } 80 \%, \text { CMd_ } 10 \% \text {, } \\
\text { CMd_ } 10 \%\end{array}$ & 1205 & $\begin{array}{l}\text { RANKER, DIS REGOLITIČNI } 80 \% \text {, DIS RJ / MAGMATSKIH kam tip } \\
\text { pl_10\%, DIS RJ / PIROKLASTIČNIH kam tip pl_10\% }\end{array}$ \\
\hline $\mathrm{CMe}$ & Eutric Cambisol & CMe_80\%, LPm_20\% & 1246 & $\begin{array}{l}\text { EVT RJ / DELUVIJU, tip 80\%, RENDZINA / POBOČNEM GRUŠČU, } \\
\text { SPRSTENINASTA_20\% }\end{array}$ \\
\hline CMd & Dystric Cambisol & CMd_70\%,CMd_30\% & 1248 & $\begin{array}{l}\text { DIS RJ / PERMO-KARBONSKIH skril + pešč tip 70\%, DIS RJ / PERMO- } \\
\text {-KARBONSKIH skril + pešč IZPRANA_30\% }\end{array}$ \\
\hline $\mathrm{CMe}$ & Eutric Cambisol & CMe_80\%, LPe_20\% & 1266 & $\begin{array}{l}\text { EVT RJ / RAZLIČNIH BAZIČNIH kam tip } 80 \% \text {, RANKER, EVT } \\
\text { REGOLITIČNI_20\% }\end{array}$ \\
\hline CMd & Dystric Cambisol & $\begin{array}{l}\text { CMd50\%, CMd_30\%, } \\
\text { CMd_20\% }\end{array}$ & 1334 & $\begin{array}{l}\text { DIS RJ / METAMORFNIH kam tip 50\%, DIS RJ / KREMENOVEM KERA- } \\
\text { TOFIRJU/ PORFIRJU, tip_30\%, DIS RJ / DIABAZU, tip_20\% }\end{array}$ \\
\hline
\end{tabular}




\begin{tabular}{|c|c|c|c|c|}
\hline $\mathrm{CMe}$ & Eutric Cambisol & $\begin{array}{l}\text { CMe60\%, LPe_20\%, } \\
\text { CMd_20\% }\end{array}$ & 1340 & $\begin{array}{l}\text { EVT RJ / MEŠANIH karb + nekarb kam tip sr gl 60\%, RANKER, EVT RE- } \\
\text { GOLITIČNI_20\%, DIS RJ / PERMO-KARBONSKIH skril + pešč tip pl }\end{array}$ \\
\hline LPm & Mollic Leptosol & LPm_80\%, CMe_20\% & 1341 & $\begin{array}{l}\text { RENDZINA / MORENI, SPRSTENINASTA } 80 \% \text {, EVT RJ / MORENI, tip } \\
\text { pl_20\% }\end{array}$ \\
\hline X & X & LPk_50\%, CMx_50\% & 1394 & RENDZINA / dol SPRSTENINASTA 50\%, RJ POKARB / dol tip_50\% \\
\hline CMd & Dystric Cambisol & $\begin{array}{l}\text { CMd60\%, LPd_20\%, } \\
\text { CMd_20\% }\end{array}$ & 1404 & $\begin{array}{l}\text { DIS RJ / FILITOIDNIH skril tip gl } 60 \% \text {, RANKER, DIS REGOLITIČ- } \\
\text { NI_20\%, DIS RJ / METAMORFNIH kam tip_ } 20 \%\end{array}$ \\
\hline $\mathrm{CMe}$ & Eutric Cambisol & $\begin{array}{l}\text { CMe60\%, LPe_20\%, } \\
\text { CMe_20\% }\end{array}$ & 1430 & $\begin{array}{l}\text { EVT RJ / MEŠANIH karb + nekarb kam tip 60\%, RANKER, EVT REGO- } \\
\text { LITIČNI_20\%, EVT RJ / MEŠANIH karb + nekarb kam IZPRANA_20\% }\end{array}$ \\
\hline $\mathrm{CMe}$ & Eutric Cambisol & CMe_80\%,CMe_20\% & 1433 & $\begin{array}{l}\text { EVT RJ / POBOČNEM GRUŠČU, tip pl 80\%, EVT RJ / POBOČNEM GRU- } \\
\text { ŠČU, tip sr g1_20\% }\end{array}$ \\
\hline $\mathrm{CMe}$ & Eutric Cambisol & CMe_80\%,CMe_20\% & 1434 & $\begin{array}{l}\text { EVT RJ / LED DOB prod + pešč NASUTINAH REK + REČ VRŠAJU, tip } \\
\text { pl } 80 \% \text {, EVT RJ / LED DOB prod + pešč NASUTINAH REK + REČ VR- } \\
\text { ŠAJU, tip }\end{array}$ \\
\hline LPm & Mollic Leptosol & LPm_60\%, LPm_40\% & 1441 & $\begin{array}{l}\text { RENDZINA / LED DOB prod + pešč NASUTINAH REK + REČ VRŠAJU, } \\
\text { SPRSTENINASTA } 60 \% \text {, RENDZINA / LED DOB prod + pešč NASUTI- } \\
\text { NAH REK + REC }\end{array}$ \\
\hline $\mathrm{CMe}$ & Eutric Cambisol & CMe_80\%, CMe_20\% & 1442 & $\begin{array}{l}\text { EVT RJ / aluv -koluv NANOSU, KOLUVIALNA 80\%, EVT RJ / aluv -koluv } \\
\text { NANOSU, tip sr g1_20\% }\end{array}$ \\
\hline $\mathrm{CMe}$ & Eutric Cambisol & CMe_70\%, CMd_30\% & 1473 & $\begin{array}{l}\text { EVT RJ / MIOCENSKIH pes pešč kongl tip 70\%, DIS RJ / MIOCENSKIH } \\
\text { pes pešč + kongl tip_30\% }\end{array}$ \\
\hline $\mathrm{CMe}$ & Eutric Cambisol & CMe_80\%, ATa_20\% & 1474 & $\begin{array}{l}\text { EVT RJ / MIOCENSKIH pes pešč kongl tip 80\%, RIGOLANA, VINOGRA- } \\
\text { DNIŠKA (VITISOL), EVT_ } 20 \%\end{array}$ \\
\hline CMd & Dystric Cambisol & CMd_70\%, LPd_30\% & 1476 & DIS RJ / METAMORFNIH kam tip 70\%, RANKER, DIS EROZIJSKI_30\% \\
\hline $\mathrm{CMe}$ & Eutric Cambisol & $\begin{array}{l}\mathrm{CMe} 40 \%, \mathrm{CMe} \_30 \% \text {, } \\
\mathrm{CMe} \_30 \%\end{array}$ & 1497 & $\begin{array}{l}\text { EVT RJ / LED DOB prod + pešč NASUTINAH REK + REČ VRŠAJU, tip g1 } \\
40 \%, \text { EVT RJ / LED DOB prod + pešč NASUTINAH REK + REČ VRŠAJU, } \\
\text { IZPRA }\end{array}$ \\
\hline $\mathrm{CMe}$ & Eutric Cambisol & CMe_60\%, LPm_40\% & 1504 & $\begin{array}{l}\text { EVT RJ / MORENI, tip pl 60\%, RENDZINA / MORENI, SPRSTENINASTA } \\
40 \%\end{array}$ \\
\hline $\mathrm{LVh}$ & Haplic Luvisol & LVh_60\%, LVh_40\% & 1505 & $\begin{array}{l}\text { IZPRANA (LUVISOL) / KONGLOMERATU, PSEVDOOGLEJENA } 60 \% \text {, } \\
\text { IZPRANA (LUVISOL) / KONGLOMERATU, tip_ } 40 \%\end{array}$ \\
\hline $\mathrm{CMe}$ & Eutric Cambisol & $\mathrm{CMe} \_80 \%, \mathrm{CMe} \_20 \%$ & 1510 & EVT RJ / SIVICI, tip 80\%, EVT RJ / SIVICI, IZPRANA_20\% \\
\hline LPm & Mollic Leptosol & LPm_60\%, LPm_40\% & 1515 & $\begin{array}{l}\text { RENDZINA / LED DOB prod + pešč NASUTINAH REK + REČ VRŠAJU, } \\
\text { SPRSTENINASTA } 60 \% \text {, RENDZINA / LED DOB prod + pešč NASUTI- } \\
\text { NAH REK + REC }\end{array}$ \\
\hline $\mathrm{CMd}$ & Dystric Cambisol & CMd_70\%,CMe_30\% & 1521 & $\begin{array}{l}\text { DIS RJ / NENEKARB FLIŠU + DEKALCIFICIRANEM LAPORJU, tip } \\
70 \% \text {, EVT RJ / DELUVIJU, tip_30\% }\end{array}$ \\
\hline FLc & Calcaric Fluvisol & FLc_70\%, FLc_30\% & 1527 & $\begin{array}{l}\text { OBREČNA, KARB sr gl / ILOVNATEM ALUVIJU70\%, OBREČNA, KARB } \\
\text { GLOBOKO OGLEJENA/ PEŠČENO PRODNATEM ALUVIJU_30\% }\end{array}$ \\
\hline $\mathrm{CMd}$ & Dystric Cambisol & $\begin{array}{l}\text { CMd } 60 \% \text {, CMd_30\%, } \\
\text { LPd_10\% }\end{array}$ & 1781 & $\begin{array}{l}\text { DIS RJ / BIOTITNO-MUSKOVITNEM BLESTNIKU, tip SR gl 60\%, DIS } \\
\text { RJ / BIOTITNO-MUSKOVITNEM BLESTNIKU, tip g1_30\%, RANKER, } \\
\text { DIS REGO }\end{array}$ \\
\hline $\mathrm{CMd}$ & Dystric Cambisol & $\begin{array}{l}\text { CMd60\%, CMd_20\%, } \\
\text { CMd_20\% }\end{array}$ & 1816 & $\begin{array}{l}\text { DIS RJ / GNAJSU, tip pl 60\%, DIS RJ / FILITOIDNIH skril tip pl_20\%, DIS } \\
\text { RJ / DIABAZU, tip pl_20\% }\end{array}$ \\
\hline $\mathrm{CMd}$ & Dystric Cambisol & $\begin{array}{l}\text { CMd } 60 \%, \text { LPd_20\%, } \\
\text { CMd_20\% }\end{array}$ & 1817 & $\begin{array}{l}\text { DIS RJ / TONALITU, tip pl } 60 \% \text {, RANKER, DIS RJAV_20\%, DIS RJ / TO- } \\
\text { NALITU, KOLUVIALNA_20\% }\end{array}$ \\
\hline
\end{tabular}

Source (Vir): Ministrstvo za kmetijstvo, gozdarstvo in prehrano. 